\title{
Historische Aspekte der Urbanisierung: Forschungsstand und Probleme
}

\author{
Teuteberg, Hans Jürgen
}

First published in:

Urbanisierung im 19. und 20. Jahrhundert, Historische und Geographische Aspekte, S. 2 - 34, Köln 1983

Münstersches Informations- und Archivsystem multimedialer Inhalte (MIAMI) URN: urn:nbn:de:hbz:6-96449464194 
HISTORISCHE ASPEKTE DER URBANISIERUNG: FORSCHUNGSSTAND UND PROBLEME

$$
\text { Von Hans J. Teuteberg }
$$

Dieser Sammelband ist unter das Leitthema „Urbanisierung im 19. und 20. Jahrhundert - historische und geographische Aspekte“ gestellt worden. Damit soll zum Ausdruck gebracht werden, daß das in den letzten einhundert Jahren quantitativ wie qualitativ kulminierende Phänomen der Verstädterung Objekt einer zeitlich wie räumlich vergleichenden Analyse bildet. Das Problem der Stadt im Industriezeitalter wird heute von der speziellen Wissenschaft der Urbanistik erforscht, die sich in Wahrheit aber noch aus einer Vielzahl unverbundener herkömmlicher Disziplinen rekrutiert. Bei allen Untersuchungen muß daher auf die Resultate verschiedener Fächer zurückgegriffen werden. Wenn hier Geographen und Historiker gemeinsam auftreten, dann stellt dies nur eine der möglichen Kooperationsformen dar. Angesichts solcher interdisziplinärer Bemühungen darf nicht überraschen, wenn viele Einzelfragen der Urbanisierung beide Fächer gleich stark berühren. Zu nennen wären hier beispielsweise die zentralörtlichen Funktionen der Städte in Abhängigkeit von ihrer Lage und Größe, die geographischen Determinanten des Bevölkerungswachstums und der Mobilität, die Standortwahl von Unternehmen im Hinblick auf natürliche Ressourcen, Arbeitskräftepotential und Verkehrsverbindungen, die soziale Segregation bei der City- und Stadtteilbildung und anderes mehr. Die unterschiedlichen Anliegen von Geschichte und Geographie liegen offensichtlich weniger in der spezifischen Auswahl der zu untersuchenden Sachverhalte als vielmehr in den Forschungsstrategien und generellen Erklärungsversuchen, die ihrerseits auf verschiedenen Wissenschaftstraditionen aufbauen. Zweck dieses Sammelbandes soll es sein, anhand einheitlicher Themenkomplexe gemeinsame wie trennende Forschungsansätze herauszuarbeiten und sich über den jeweils erreichten Erkenntnisstand besser als zuvor gegenseitig ins Bild zu setzen. Verständlicherweise können wie immer bei solchen Anlässen nur einige spezifisch ausgewählte Problembereiche intensiver diskutiert werden, die beide Fächer zugleich beschäftigen Auf den ersten Blick mag es scheinen, als würden die Themen dieses Bandes etwas unverbunden nebeneinanderstehen. Eine nähere Beschäftigung mi ihnen wird aber schnell zeigen, wie mannigfach verzahnt sie in Wirklichkeit sind. Das Feld der Urbanisierungsforschung ist sehr viel komplexer als man früher anzunehmen geneigt war.

Angesichts eines multidisziplinären wie auch internationalen Gedankenaustauschs erscheint es besonders dringlich, zunächst begriffliche Verständigungsversuche zu unternehmen. Der hier im Brennpunkt stehende Terminus „Urbanisierung“ ist wie seine Synonyme „Urbanisation“ und „Verstädterung“ (engl. urbanization, franz. urbanisation) weit, vielschichtig und mehrdeutig, so $\mathrm{da}$ eine genuin akzeptable Definition nicht angeboten werden kann. Seitdem vermutlich Karl Bücher 1893 den Begriff Urbanisierung erstmals im heutigen Sinne in die wissenschaftliche Debatte einführte, haben sich eine Fülle von Ausdeutungen ergeben, die sich nur schwer auf einen Nenner bringen lassen 1 . Das Spektrum der Erklärungsversuche reicht von quantitativ-statistischen Zugriffen der demographischen Entwicklung und siedlungsgeographischen bzw. sozialtopographischen Strukturveränderungen über Beiträge zur Theorie des Wirtschaftswachstums und sozialen Wandels bis zu sozialpsychologischen Begriffsbestimmungen. Aufgabe dieses einleitenden Beitrages ist es, einen kurzen Aufriß zu geben, inwieweit sich die Historiker im weiteren Sinne in den letzten einhundert Jahren um die Aufheilung des Verstädterungsphänomens bemüht haben und welche Problemfelder und Forschungsmethoden $\mathrm{z} . \mathrm{Zt}$ hauptsächlich diskutiert werden. Dabei muß auch auf die Erkenntnisfortschritte benachbarter Fächer, insbesondere der mehr generalisierenden Wirtschafts- und Sozialwissenschaften, zurückgegriffen werden. Dies darf als durchaus legitim angesehen werden, da die moderne Stadtgeschichte ähnlich wie die Urbanistik selbst eine interdisziplinäre Vorgeschichte hat.

Wann hat die stadtgeschichtliche Forschung begonnen? Erste Beschäftigungen mit dem sich wandelnden Bild der Stadt sind so alt wie die Geschichte der menschlichen Zivilisation, so daß es müßig erscheint, nach irgendwelchen näher datierbaren Ursprüngen zu fahnden. Jahrtausendelang wurde Stadtgeschichte ganz offensichtlich nur als integraler Bestandteil mythisch-religiös überhöhter Heils- und Weltgeschichte begriffen. Erst mit dem Auftauchen der modernen Staatswissenschaften im Zeichen des Naturrechts, der Renaissance und besonders dann der Aufklärung begannen im Rahmen der sich herausbildenden Kameralistik tastende Versuche, die Erscheinung der Stadt im Gegensatz zum „platten Land“ erstmals gedanklich abstrahiert zu erfassen, wobei man sich zunächst an rein äußerlichen Merkmalen orientierte wie z.B. dem Vorhandensein einer Mauer oder eines Stadttores, der Zahl der Bewohner und ihrem Handel und Gewerbe bzw. der Existenz eines siegelführenden Stadtrates. Als besonderes Charakteristikum zur Differenzierung von Stadt und Land wurde die Verleihung eines Marktrechtes durch den jeweiligen Landesherrn angesehen ${ }^{2}$. Die Antwort, was unter einer Stadt zu verstehen sei, mußte bei einer solchen Addition von äußerlichen Charakteristika noch recht disparat ausfallen, kam es doch ganz darauf an, ob man eine Stadt vom Standpunkt der Fortifikation, der wirtschaftlichen Versorgung der Bevölkerung, der Staatssteuern oder aber von der Baukunst her betrachtete. Die Enzyklopädien und Lexika des 18.Jahrhunderts sind voller Beispiele für solche Betrachtungsweisen.

' Bücher erklärte den Begriff Urbanisierung mit „Verstadtlichung der Kulturmenschheit". Vergl. KARL BÜCHER, Großstadttypen aus fünf Jahrtausenden, in: DERS, Die Entstehung der Volkswirtschaft, 1, 14.-15.Aufl. Tübingen 1920, S. 386.

2 Die deutsche Kameralistik definierte das Wesen der Stadt wie folgt: „Die Stadt ist ein Zusammenhang von Gesellschaften, Familien und einzelnen Personen, "die in einem verwahrten Orte unter Aufsicht und Direktion eines Polizey-Collegii, welches man einen Stadtrath nennet... bei einander wohnen, um mit desto besserem Erfolge, Wirkung und Zusammenhänge solche Gewerbe und Nahrungsarten zu betreiben, die sowohl der Landes-Nothdurft und Bequemlichkeit, als zu der Verbindung des gesamten Nahrungsstandes im Lande erforderlich waren“. JoHANN-HeinRICH GotTLIEB JUSTI, Staatswirthschaft, Theil 1, Kopenhagen $1758, \$ 457$. Zum Problem der Stadtforschung im 18.Jh. vergl. FRANKLIN KoPITSCH (Hrsg.), Aufklärung, Absolutismus und Bürgertum in Deutschland, München 1976 (Einl.). 
Wichtig für den Beginn einer autonomen Stadtgeschichtsschreibung war offensichtlich die aufkommende Bevölkerungs-, Medizinal- und Wirtschaftsstatistik. An englische und französische Vorbilder sich anlehnend stellte man aus Kirchenregistern ausgezogene Geburts-, Heirats- und Sterbedaten bei der Bevölkerung von Stadt und Land vergleichend gegenüber, wobei erste dynamische Entwicklungsstrukturen städtischer Gemeinwesen ins Blickfeld gerieten ${ }^{3}$. Die Auseinandersetzung mit der „Politischen Arithmetik“ und malthusianischen Bevölkerungstheorie, die Gründung von Statistischen Bureaus und Statistischen Vereinen in den deutschen Bundesstaaten sowie dann von Statistischen Ämtern in größeren deutschen Städten und schließlich die einsetzende Reichsstatistik mit ihren verschiedenen Zweigen haben wesentliche Momente der einsetzenden Verstädterung Deutschlands sowohl zahlenmäßig messend wie verbal deskriptiv erstmals ins allgemeine Bewußtsein gebracht 4 . Der Zusammenschluß der deutschen Städtestatistiker zu regelmäßigen Konferenzen ab 1879, die Herausgabe des „Statistischen Jahrbuchs deutscher Städte“ durch W. Neefe ab 1890 sowie der Beginn international vergleichender Großstadtstatistik ab 1876 haben die Kommunalstatistik in wenigen Jahrzehnten zu einem verlässlichen Instrument der Wissenschaft gemacht und das erste brauchbare Fundament für eine Quantifizierung geschaffen 5 .

Um eine „Morphologie der Großstadtbevölkerung“ zu schaffen, wie es der bayerische Statistiker Georg von Mayr nannte, wurde die städtische $\mathrm{Be}$ völkerung nach Alter, Geschlecht und Beruf gegliedert und mit dem Ausmaß der Zuwanderung in die Städte in Beziehung gesetzt. Die damals empirisch erarbeiteten Populations- und Wanderungsstatistiken müssen als erste wichtige Bausteine für die quantitativ arbeitende Urbanisierungsforschung angesehen werden 6 . Der Amerikaner Adna Ferrin Weber hat die Resultate der

${ }^{3}$ So unterzog der Feldprediger Friedrichs d.Gr., Johann Peter Süßmilch, die Sterblichkeit in preußischen Städten einer ersten zusammenfassenden Analyse, wobei er zu dem Ergebnis kam, $\mathrm{da} ß$ diese mit der Größe relativ zunehme, während die Heiratsquote sinke. Das Leben in den Städten nannte er vom populationistischen Standpunkt „fast einen Pestschaden“. JoHANN PETER SÜMILCH, Göttliche Ordnung in der Veränderung des menschlichen Geschlechts, aus der Geburt, dem Tode und der Fortpflanzung desselben erwiesen, 2. ganz umgearbeitete Aufl., Berlin 1761. Vergl. Ders., Gedancken von den epidemischen Krankheiten und dem größere Sterben des 1757ten Jahres, in einem Sendschreiben an die Herren Verfasser der Göttingschen Anzeigen von gelehrten Sachen auf derselben Verlangen entworfen, Berlin 1758.

fast immer auch mit kommunalen rungslehre, Jena 1929, S. 108. Die Rezeption der befaßte, vergl. PAUL MoMBERT, Bevölkeaufgearbeitet bei KLAUS JǗRGEN MATZ, Pauperismus und Bevölkerung Die Ehebeschränkungen in den süddeutschen Staaten während des 19.Johrung. Die gesetzlichen - Gute statistische Gegenüberstellungen von Stadt- und Landbevölkerung briat 1980. anderem GEORG VON VIEBAHN, Statistik des zollvereinten und nördlichen Deutsch bringt unter Berlin 1858-1868.

5 Das „Statistische Jahrbuch Deutscher Städte“ ist für die Geschichte der Urbanisierung eine unerschöpfliche Fundgrube, die bisher von Historikern noch wenig ausgenutzt wurde. Die Entwicklung aller größeren und mittleren deutschen Stadtgemeinden, die über ein Statistisches Amt verfügten, kann dort im einzelnen verfolgt werden. Unter anderem finden sich dort

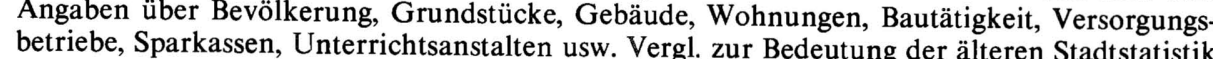
ELISABETH PFEIL, Großstadtforschung, 2.neu bearb. Aufl. Hannover 1972, S. 33. Die drei Bevölkerungsstufen, München 1889. - H. RAUCHBERG, Die Bevölkerung Österreichs ersten Kommunalstatistik, die etwa zur gleichen Zeit auch in anderen entwickelten Volkswirtschaften begonnen worden war, in einem bis heute immer wieder zitierten klassischen Werk zusammengefaßt und damit die Bedeutung wieder zitierten klassischen Werk zusammengefaßt und damit die Bedeutung
dieser quantitativen Forschungsansätze für die vergleichende Städteforschung unterstrichen 7 . Webers mühseliger Versuch, die unterschiedlich angefertigten Statistiken aus verschiedenen Ländern Europas und den USA in einen einheitlich interpretierbaren Rahmen zu zwängen, mußte verständlicherweise noch unvollkommen bleiben und löste berechtigte Kritik aus. Sein Buch machte aber weithin deutlich, daß es sich bei der Urbanisierung um ein weltweites, offenbar mit der Industrialisierung und Modernisierung eng zusammenhängendes Strukturproblem handelte und die damals viel erörterte Soziale Frage" zugleich bis zu einem gewissen Grade als eine Frage der Verstädterung angesehen werden mußte. Die schnell wachsenden großen Städte brachten überall darüber hinaus eine Fülle von rechtlich-politischen und wirtschaftlich-technischen sowie sozialpsychologischen und kultur-kritischen Problemen ins Spiel, die von den Städtestatistikern noch nicht beantwortet werden konnten.

Eine weitere wichtige Unterstützung erfuhr die Urbanisierungsforschung durch die im Zuge der politischen Romantik und des Historismus aufkommende Historische Rechtsschule. Seit etwa der Mitte des 19.Jahrhunderts ist unter ihren Anstößen ein nahezu ständig fließender Strom rechts- und verfassungsgeschichtlicher Studien entstanden, die sich in der Hauptsache allerdings zunächst mit der spätmittelalterlichen bzw, frühneuzeitlichen Stadt befaßten. Die Forschungen von Wilhelm Arnold, Georg Ludwig von Maurer und Karl Theodor von Inama-Sternegg, aber auch von Rudolph Gneist, Hugo Preuß, Rudolph Sohm, Friedrich Keutgen und besonders von Georg von

auf Grund der Ergebnisse der Volkszählung von 1890, Wien 1895. - RichARD KUCZYNSKI, De Zug nach der Stadt, Stuttgart 1897. - CARL BALLOD, Die Lebensfahigkeit der stådtischen und ländlichen Bevölkerung, Leipzig 1897. - H.BLEICHER, Die Bewegung der Bevölkerung im Jahre 1891, insbesondere Studien über Wanderungen, durchgeführt an der Stadt Frankfurt am Main in: Beiträge zur Statistik der Stadt Frankfurt a.M., Frankfurt/M. 1893. - R.BöCKH, Der Anteil der örtlichen Bewegung an der Zunahme der Bevölkerung, in: Congrès International d'Hygiène et de Démographie, Budapest 1895. - N.BRüCKNER, Die Entwicklung der großstädtischen Bevölkerung im Gebiet des Deutschen Reiches, in: Allgemeines Statistisches Archiv, 1899. - CARL BALloD, Die mittlere Lebensdauer in Stadt und Land, Leipzig 1899. - HANS ALLENDORF, Der Zuzug in die Städte, Jena 1901. - GeORG vON MAYR, Statistik und Gesellschaftslehre. Der Stand der deutschen Städtestatistik, in: Allgemeines Statistisches Archiv, 1903 (6. Erg.heft). - SiEGMUND SCHOTT, Das Wachstum der deutschen Großstadte seit 1871, in: die Agclomerationsverbältnisse der Bevölkerung im Köniorich Bayern München 1904. SIEGMUND SCHOTT, Die Citybildung in deutschen Großstän Bait 1871, in: Statistisches Jahrbuch deutscher Städte, 14,1907, S. 21-32 - M. BROESIKE, Arbeitsort und Wohnort der Bevölkerung in den Großstädten und S. 21-32 Industrieziren Preußens am 1.Dez. 1900 der Zeitschrift des Kgl.preußischen Statistischen Büros, 44, 1907, S. 492. - Ders., Die Binnenwanderung im preußischen Staate nach Kreisen 1895-1900, in: Zeitschrift des Kol.preussischen Statischen Büros 42, 1902, S. 273-298. - SiEGMUND SCHOTT, Die großstädtischen Agglomerationen des Deutschen Reiches 1871-1910, Breslau 1912. - GotTLIEB GASSERT, Die berufliche Struktur der deutschen Großstädte nach der Berufszählung von 1907, Diss. Heidelberg 1917. - W. FranKE, Die Volkszahl deutscher Städte Ende des 18. und Anfang des 19.Jahrhunderts, in: Zeitschrift des Preußischen Statistischen Landesamtes Berlin, 62, 1922. ADNA FerRIN Weber, The Growth of the Cities in the Nineteenth Century: A Study in Statistic (1899), Neudruck Ithaca, N.Y., 1967. 
Below haben hier die institutionellen Anfänge der frühesten Verstädterung Deutschlands anhand der Quellen herausgearbeitet, um nur einige der wichtigsten Gelehrtennamen zu nennen 8.

Die ältere Historische Schule der Nationalökonomie war unter dem Eindruck der massenhaft einsetzenden Stadtwanderungen im späten 19. Jahrhundert ebenfalls stark an dem Problem der Verstädterung interessiert. Sie lenkte den Blick aber öfter als die Juristen auch auf die eigene Gegenwart. So untersuchte, historisch allerdings meistens weit ausholend, Wilhelm Roscher die Getreide-, Finanz- und Armenpolitik der Städte, Bruno Hildebrand setzte sich nach einer Englandreise mit Friedrich Engels 1845 verfaßten düsteren Gemälde der aufkommenden englischen Industriestädte im Hinblick auf die deutsche Situation auseinander, und Karl Knies machte die Wirkung des aufkommenden Eisenbahnsystems und der Geldwirtschaft auf das Städtewachstum erstmals zum Gegenstand von Analysen 9. Die sich im letzten Drittel des vorigen Jahrhunderts dann vor allem im „Verein für Socialpolitik“ formierende jüngere Historische Schule der Wirtschaftswissenschaft setze diese Untersuchungen in wesentlich verbreiterter Form fort, wobei auf die richtungsweisenden Arbeiten von Georg Friedrich Knapp, Gustav Schmoller, Gustav Schönberg, Richard Ehrenberg und Adolph Wagner vor Ausbruch des 1. Weltkrieges besonders hinzuweisen ist, die ihrerseits wiederum eine Reihe von Schülern zu lokalgeschichtlichen Vertiefungen anregten ${ }^{10}$. Die Studien

8 Wilhelm ARNold, Verfassungsgeschichte deutscher Freistädte, 2 Bde., Gotha 1854. - GeORG LUDWIG RITTER VON MAURER, Geschichte der deutschen Gemeindeverfassung in Einzeldarstellungen, 12 Bde., Leipzig 1854-1871. - K.H. ROTH VON SCHRECKENSTEIN, Das Patriziat in den Stadten, 1856. - F. BARTHOLD, Geschichte der deutschen Stadte und des deutschen zurrgertums, 4 Teile in 2 Bden., 2. Aufl., Leipzig 1859 (Nachdruck 1976). - G. ECKERTZ, Quellen des Eigentums in deutschen Städten, Basel 1861. - L. ENNEN, Geschichte der Stadt Köln, 5 Bde., Köln 1863-1880. - GEORG LuDWIG RITTER vON MAURER, Geschichte der Städteverfassung in Deutschland, 4 Bde., Erlangen 1869-1871. - GeORg voN BeLow, Die Entstehung des deutschen Städtewesens (Festschrift), Leipzig 1890. - Sebastian SchwarZ, Anfänge des Städtewesens in Elbe-und Saalegegenden, Leipzig 1892. - Siegfried RitsCHL, Markt und Stadt in ihrem rechtlichen Verhältnis, Leipzig 1897.- KARL THEODOR vON INAMA-STERNEGG, Deutsche Wirtschaftsgeschichte, Leipzig 1901. - RUDOLPH SOHM, Institutionen. Ein Lehrbuch der Geschichte und des Systems des Privatrechts, 10. Aufl., Leipzig 1901. - Friedrich Keutgen, Ämter und Zünfte, Jena 1903. - Hugo Preuss, Die Entwicklung des deutschen Städtewesens, Bd.1: Entwicklung der deutschen Städteverfassung, Leipzig 1906. - GEORG vON BELOW, Das ältere deutsche Städtewesen und Bürgertum, Leipzig 1898 (2. Aufl. 1905, 3. Aufl. 1922). DERS., Territorium und Stadt. Aufsätze zur deutschen Verfassungs-, Verwaltungs- und Wirtschaftsgeschichte, München 1900 (2. Aufl. 1923). Vergl. zu diesem Komplex der älteren Stadgeschichtsforschung die immer noch hervorragend informierende Zusammenfassung von alwissenschaften, 9, Tübingen-Göttingen 1956, S. 780-785.

9 WILHEI ROCCHER Über Kornhandel und Teu. - DERS. System der Finanzwirtschaft (System der Volkswirtschaft, Bd 4), Stuttgart 1886 . Ders., System der Armenpflege und Armenpolitik, Stuttgart 1894. - FrifDrict ENGELS, Die DeRS., System der Armenpflege und Armenpolitik, Stuttgart 1894. - FrIEDRICH ENGELS, Die tionalökonomie der Gegenwart und Zukunft, Frankfurt a.M. 1848. Neu hrsg. von Heinrich Waentig, Jena 1922. - KARL KNIES, Die Eisenbahn und ihre Wirkungen, Braunschweig 1853. Ders., Geld und Kredit, 2 Bde., Berlin 1873-1879 (2. Aufl. 1885).

10 GeORG FrIEDRICH KNaPp, Über die Ermittlung der Sterblichkeit aus den Aufzeichnungen der Bevölkerungsstatistik, Leipzig 1868. - Gustav Schmoller, Straßburg zur Zeit der Zunftkämpfe, Straßburg 1875. - GuSTAV SCHÖNBERG, Finanzverhătnisse der Stadt Basel, Tübingen 1879. - GuSTAV SChMOLleR, Die Straßburger Tucher- und Weberzunft, Straßburg 1879. EUGEN NüBLING, Ulms Baumwollweberei im Mittelalter, Leipzig 1890. - RICHARD EHRENBERG, bewegten sich ähnlich wie die der vergleichenden Rechts- und Verfassungsbew geschichte teils im ausgehenden Mittelalter, teils

Dem Nationalökonom und Historiker Karl Bücher gebührt das unbestreitbare Verdienst, die vielfältigen Forschungsergebnisse zur allgemeinen Stadtentwicklung wie auch zu dem speziellen Großstadtphänomen erstmals zucenten aus den Quellen erarbeitete und methodisch sammerordentlich anregende Untersuchung „Die Bevölkerung Frankfurt am außerordentlich anregende Untersuchung „Die Bevölkerung Frankfurt am deutsamen Abhandlungen in seinem immer wieder aufgelegten Sammelwerk Die Entstehung der Volkswirtschaft" (1893) weisen ihn ähnlich etwa wie "D. Legoyt und E.Levasseur in Frankreich als ersten speziellen Urbanisierungsforscher aus 11 . Unter anderem setzte er sich hier mit den Großstadttypen rungsforscher aus 11 . Unter anderem letzten fünf Jahrtausende, mit der sozialen Schichtung in einer im Laufe der letzten fünf Jahrtausende, mit der sozialen Schichtung in einer kommunalen Aufgabenbestandes auseinander. In selten gelehrter Meisterschaft verstand es Bücher, die Besonderheiten der modernen Megapolis durch Vergleiche mit der antiken und mittelalterlichen Stadt strukturell abstrahierend herauszuarbeiten, die in ihren damaligen Zeiten durchaus den Charakter einer "Großstadt" hatten. Die neuere „Verstadtlichung der Kultur", wie er die Urbanisierung erstmals definierte, wurde als eine unausweichliche Folge ökonomischer und gesellschaftlicher Transformationsprozesse interpretiert und die damals beliebte Verurteilung des "Zuges zur Stadt" logischerweise scharf abgelehnt 12. Bücher, zunächst Lehrer und dann Journalist gewesen, besaß ein ungewöhnliches Gespür, zeitgenössische Probleme in eine größere historische Entwicklung zu stellen und von daher neu zu beleuchten, wobei auch die Statistik herangezogen wurde. Seine betont optimistische Sicht der Verstädterung richtete sich gegen eine im späten 19. Jahrhundert weit verbreitete und emotionell tief verwurzelte Großstadtfeindschaft. Schon die romantische Staatslehre und die Frühsozialisten verschiedener Richtungen

Das Zeitalter der Fugger. Geldkapital und Kreditverkehr im XIV. Jahrhundert, 2 Bde., Jena 1898. - Alors SCHULTE, Geschichte des mittelalterlichen Handels und Verkehrs zwischen Westdeutschland und Italien mit Ausschluß Venedigs, 2 Bde., Leipzig 1900. - ALEXANDER Dietz, Frankfurter Handelsgeschichte, 4 Bde., Frankfurt a.M. 1910. - Wilhelm Franz, Bilder aus der Geschichte des deutschen Städtewesens, Berlin 1910. - Eine Fülle von Abhandlungen zur Wirtschaftsgeschichte des deutschen Städtewesens in älterer Zeit wie zur Urbanisierung des 19. Jahrhunderts dürfte in den Zeitschriften enthalten sein, die Zon den historisch orientierte Nationalökonomen herausgegeben wurden. Zu nennen sind hier vor allem das Jahrbuch fü Geir die Statistik (1863 ff) und das Thünen-Archiv (1906-1922).

KaRL BÜCHER,
Tübingen 1920 .

12 Büchers stadtgeschichtliche Studien hängen auf das engste mit seiner zugleich aufgestellten wirtschaftlichen Stufentheorie Hauswirtschaft-Stadtwirtschaft-Volkswirtschaft zusammen. Vergl. KARL BüCHER, Volkswirtschaftliche Entwicklungsstufen, in: Grundriß der Sozialökonomik, 2. Aufl., Tübingen 1924. - Bücher machte hier unter anderem darauf aufmerksam, daß es sich bei den ersten Stadtbildungen um freiwillige Siedlungszusammenschlüsse gehandelt habe. Die moderne Großstadtbildung stehe dagegen unter dem Druck der ländlichen Übervölkerung und bilde daher ein notwendiges Ventil für die wirtschaftliche und soziale Weiterentwicklung einer Kultur. 
hatten in der ersten Hälfte des 19. Jahrhunderts die aufkommenden großen urbanen Agglomerationen ebenso wie die Zentralisierung des gewerblichfabrikatorischen Produktionsprozesses als Symbole des heraufziehenden "Mammonismus" bzw. der "Geldaristokratie“" und als gefährliche Atomisierung der ständisch korporierten alten Gesellschaft begriffen sowie als Auswüchse eines entseelten „Maschinenzeitalters“ und Brutstätten einer allgemeinen Pauperisierung und Proletarisierung diskreditiert ${ }^{13}$. Im Mittelpunkt der allgemeinen Gesellschafts- und Kulturkritik stand verständlicherweise das neue Massenwohnungselend in den großen Industriestädten. Die vormärzliche Armutliteratur in Deutschland hatte sich noch wie in den Jahrhunderten zuvor fast nur mit ländlichen und kleinstädtischen Verhältnissen beschäftigt. Nur einige wenige Ortsbeschreibungen aus den Federn städtischer Armenärzte deuteten in vagen Umrissen und Ausschnitten die nun heraufziehenden hygienischen Fragen der neuen industriellen Verstädterung an ${ }^{14}$. Die sozialen Fragen des eigentlichen Verstädterungsprozesses blieben dabei noch verborgen.

Als aber in den dreißiger und vierziger Jahren des 19. Jahrhunderts in England, Frankreich und Belgien aufgrund von ersten Umfragen die in dustrielle „Wohnungsfrage“ als Bestandteil der neuen „Arbeiterfrage“ lebhaft erörtert wurde, griff man dies auch in einigen deutschen Bundesstaaten in Form von Übersetzungen und Kommentaren auf, zumal sich die Zensur gelockert hatte. Einiges Aufsehen erregte zunächst der Barmer Fabrikantensohn Friedrich Engels mit seinem betont polemisch einseitig angelegten Buch „Die Lage der arbeitenden Klasse in England“ (1845), worin er die Arbeiterquartiere in einigen englischen Industriestädten und insbesondere in Manchester, wo er eine Zeitlang in einer Filiale des väterlichen Unternehmens gearbeitet hatte, einer scharfen Kritik unterzog. Das Buch, das Karl Marx und den aufkommenden "Wissenschaftlichen Sozialismus" sowie die Arbeiterbewegung stark beeinflußte, forderte wegen seiner Einseitigkeit und düsteren Prophezeiungen nicht nur den Marburger Nationalökonomen und Achtund-

${ }_{13}$ Diese Problematik ist bis heute wenig untersucht. Auseinandersetzungen mit der aufkommenden Industriestadt in diesem kritisch abwertenden Sinne finden sich bei J.G. FicHTE, Der geschloßne Handelsstaat, Tübingen 1800, Abdruck in: Ders., Sämtliche Werke, Bd. 3, 2. Aufl. 1925. - AdAM Müller, Elemente der Staatskunst, 3 Theile, Berlin 1809 (3. Aufl. 1926). Charles Fourier, Cités ouvriers, Paris 1849. - Vergl. Klaus BergmanN, Agrarromantik und Großstadtfeindschaft, Meisenheim/Glan 1970.

${ }^{14}$ CARL JANTKE/DIETRICH HILGER, Die Eigentumslosen, Freiburg-München 1965. - VoLKER GLäNTZER, Ländliches Wohnen vor der Industrialisierung, Münster 1980. - RUDOLF BRAUN, verlagsindustriellen Heimarbeit in einem ländlichen Industriegebiet (Zürcher Oberland) vor 1800, Winterthur 1960, S. 155-180. Vergl. die zeitgenössischen Schriften ADO PH LETTE Die Vertheilungsverhältnisse des Grundbesitzes in Preußen, in: Zeitschrift des Central-Vereins für das Wohl der arbeitenden Klassen, 2, 1860, S. 87-112. - THEODOR FREIHERR vON DER GOLTZ Ländliche Arbeiterwohnungen oder Darstellung der Nothwendigkeit einer Verbesserung der ländlichen Arbeiterverhältnisse, Königsberg 1865. - W. SENFT, Über gesundheitsmässige Einrichtung ländlicher Arbeiterwohnungen, in: Der Arbeiterfreund, 3, 1865, S. 173-186. - A FRIEDLÄNDER, Über die Wohnungsverhältnisse der ärmeren Classen der ländlichen Bevölkerung in hygienischer Beziehung, in: Deutsche Vierteljahrsschrift für öffentliche Gesundheitspflege, 9, 1877, S. 126-156. Vergl. auch ENID GAULDIE, Cruel Habitations. A History of Working-Class Housing 1780-1918, London 1974. - Karl Paul Brandelmeier, Medizinische Ortsbeschreibungen des 19. Jahrhunderts im deutschen Sprachbereich (Abhandlungen zur Geschichte der Medizin und Naturwissenschaften, H. 38), Berlin 1942.
vierziger-Politiker Bruno Hildebrand, sondern auch den bekannten preussisch-protestantischen Sozialreformer Victor Aime Huber zur Kritik heraus, nachdem auch diese die englischen Industriestädte bereist hatten 15.

Der im Anschluß an die erste deutsche Gewerbeausstellung 1844 entstandene sozialliberale „Central-Verein für das Wohl der arbeitenden Klassen in Preußen" wurde nun zum ersten öffentlichen Forum in Deutschland, auf dem man sich ausführlich und speziell mit den „Wohnungsfragen der arbeitenden Klassen", aber auch allgemeinen Problemen der Verstädterung unter meist praktischen Aspekten auseinandersetzte ${ }^{16}$. In den von dem Verein herausgegebenen Mitteilungsblättern, aus denen dann seit den sechziger Jahren die bis zum Ausbruch des 1. Weltkriegs 1914 erscheinende Zeitschrift Der Arbeiterfreund" entstand, finden sich reiche Anschauungsmaterialien über die ersten Auswirkungen der Verstädterung in Deutschland sowie erste programmatische Überlegungen zur Städteplanung und Wohnungssanierung ${ }^{17}$. Vor allem wurde hier auch in ausführlichster Form das gesamte ausländische einschlägige Schrifttum besprochen und Nutzanwendungen für die deutsche Entwicklung zu ziehen versucht. Als einer der frühesten und profiliertesten Städtereformer im Rahmen dieser Vereinsbestrebungen erwies sich der schon erwähnte Victor Aimé Huber. Nach entsprechenden Studienreisen $1854 \mathrm{im}$ benachbarten westlichen Ausland im Auftrag der preußischen Regierung machte er als erster Wissenschaftler in Deutschland in zusammenhängender Weise auf die Problematik der speziellen "Wohnungsfrage" aufmerksam, die von ihm als Ausfluß einer größeren wirtschaftlichen und gesellschaftlichen Strukturwandlung bezeichnet wurde. Die bisherigen iso-

15 Friedrich EnGels, Die Lage der arbeitenden Klasse, Leipzig 1845. (Vergl. dort das Kapitel „Die großen Städte“", S. 89ff). - BRUNO HILDEBRAND, Die Nationalökonomie der Gegenwart und Zukunft (1848), neu hrsg. von H. Waentig, Jena 1922. - VICroR ArME HuBER, Zur neuesten
Literatur, in: Janus. Jahrbücher deutscher Gesinnung, Bildung und That, Bd. 2, Berlin 1845, H.

16 JÜRGEN Reulecke, Der Centralverein für das Wohl der arbeitenden Klassen in Preußen, in: Mittheilungen des Centralvereins für das Wohl der arbeitenden Klassen (unveränderter Neudruck der Ausgabe Berlin 1848-1858 in fünf Bänden) hrsg. von W. KöLLMANN/J. ReulECKE, Bd. 1, Hagen 1980, S. 23-42

${ }^{7}$ Aus der Fülle der Themen, die auf den Versammlungen des Central-Vereins für das Wohl der arbeitenden Klassen in Preußen in den vierziger und fünfziger Jahren erörtert wurden, seien hier folgende Beispiele zitiert: GAEBLER, Idee und Bedeutung der Berliner gemeinnützigen Baugesellschaft, Berlin 1848. - W. Eммгсн, Vergleichende Bemerkungen über die Bestrebungen zur Verbesserung der Wohnungsverhältnisse der arbeitenden Klassen in verschiedenen Landern, namentlich in England, in: Zeitschrift des Central-Vereins für das Wohl der Waschanstalten, besonders in England in: Mittheilungen des Central-Vereins für das Wohl der arbeitenden Klassen N.F 3, 1851/52, H. 15, S. 1-23 - G. K NOBH Berlin nicht mehr Gebäude mit kleinen Wohnungen gebaut? in: Mittheilungen des Centralvereins für das Wohl der arbeitenden Klassen N.F., 1, 1853-1855, H. 3, S. 246-249. - W. Еммісн, Betrachtungen über Ursache und Abhülfe des Mangels an kleinen und mittleren Wohunugen in Berlin, nebst Ermittlung der Anlagekosten und Ertragsverhältnisse von Grundstücken verschiedenen Umfangs mit bürgerlicher Einrichtung, in: Ebd. S. 249-257. - DeRS Über die Kellerwohnungen in Berlin, die nachtheiligen Einflüsse derselben auf die Gesundheit der Bewohner und Vorschläge zu deren Abhülfe, in: Ebd., 2, 1856-1858, S. 218-249. - W. Еммісн, Bericht über Säuglings- und Kinderbewahr-Anstalten in baulicher Beziehung, in: Ebd., 1, 1851/52, H. 15, S. 34-51, - KoBES, Allgemeine Einrichtungen im Interesse der Arbeiter der Flachsgarn-Maschinenspinnerei und Weberei Erdmannsdorf im Jahre 1852. 4. Wohnungsverhältnisse, in: Ebd., 1, 1853-1855, H. 3, S. 219-220. 
lierten und nur an praktischen Lösungsvorschlägen orientierten Betrachtungsweisen überwindend brachte er den Kernbereich der modernen Verstädterung in einen größeren Kontext und stellte generalisierende Reflexionen darüber an 18 .

Karl Knies, der bereits erwähnte Mitbegründer der älteren Historischen Schule der Nationalökonomie, legte vor dem gleichen Gremium in den fünfziger Jahren erstmals die Bedingungen der Mietpreisbildung bei de städtischen Bevölkerung dar, wobei die sozialen Unterschichten verständlicherweise wiederum im Mittelpunkt standen. Auch der der Freihandels bewegung nahestehende „Kongreß deutscher Volkswirthe“ sah sich veranlaßt die sich verschärfende Wohnungsnot in den rasch anwachsenden Großstädten bereits 1868 zum Thema einer eigenen Tagung zu machen ${ }^{19}$. Viele Mitglieder dieser vor allem in norddeutschen Küstenstädten auftretenden Vereinigung waren zugleich im „Centralverein für das Wohl der arbeitenden Klassen“ tätig. Hatte sich dieser mehr den sozialen und sozialpolitischen Fragen der Urbanisierung zugewandt, so sah der „Kongress deutscher Volkswirthe“ vor allem sein Ziel darin, die ökonomischen Aspekte dieser drängenden Tagesfrage zu analysieren. Hier trat vor allem der Publizist und Journalist Julius Faucher hervor, der die weit verbreiteten liberalen Vorstellungen von einem automatischen Ausgleich von Angebot und Nachfrage auf dem Wohnungsmarkt anhand beobachteter Fakten widerlegte. Nachdrücklich forderte er die Heranziehung der Kulturgeschichte als empirische Wissenschaft zur Ergänzung der seiner Meinung nach relativ realitätsfernen klassischen Nationalökonomie und machte auch auf die psychologischen Momente einer wachsenden Verstädterung für das menschliche Dasein aufmerksam ${ }^{20}$. Der aus Österreich stammende Städtereformer ging interessanterweise nicht mehr wie seine Vorgänger von einer eingeschränkten „Arbeiterwohnungsfrage“ aus, sondern konstatierte in der Mitte der sechziger Jahre bereits eine „zusam menschrumpfende Räumlichkeit der Privatwohnungen aller Stände“", womit die „Wohnungsfrage“ ebenfalls als Problem des allgemeinen sozialen und

18 VICTOR AimÉ Huber, Reisebriefe aus Belgien, Frankreich und England im Sommer 1854 Hamburg 1855. - Die 50 Thesen des Professor Huber, in: Mittheilungen des Centralvereins für das Wohl der arbeitenden Klassen, N.F., 2, 1856, S. 289-293. - DeRs., Die Wohnungsnoth de kleinen Leute in großen Städten, Leipzig 1857. - DERS., Die Wohnungsfrage in Frankreich und England, in: Zeitschrift des Central-Vereins für das Wohl der arbeitenden Klassen, 2, 1860, S. 3-37 und 3, 1861, S. 123-196. - DERs. (Hrsg.), Concordia. Beiträge zur Lösung der socialen Frage in zwanglosen Heften, H. 1-8, Leipzig 1861 (Vergl. bes. H. 2 und 3). - DeRs., Über die geeignetsten Maßregeln zur Abhülfe der Wohnungsnoth, in: Der Arbeiterfreund, 3, 1865, S. 143-172. - Ders., Die Wohnungsnoth und die Privatspekulation, in: Der Arbeiterfreund, 5, 1867, S. 420-443.

19 KARL KNIES, Über den Wohnungsnothstand der unteren Volksschichten und die Bedingungen des Mietpreises, in: Zeitschrift für die gesammte Staatswissenschaft, 15, 1859, S. 83-107. - Die ständigen Deputation des Kongresses deutscher Volkswirthe, hrsg vom Centralverein für das Wohl der arbeitenden Klassen, Berlin 1865 .

20 JULIUS FAUCHER, Die Bewegung für Wohnungsreform, in: Vierteljahresschrift für Volkwirtschaft und Kulturgeschichte, 3, 1865, H. 4, S. 127-199 und ebd, 4, 1866, H. 3, S. 86-151. Ders., Vergleichende Kulturbilder (Berlin-Wien-Paris-London), Leipzig 1877. - DeRs., Die Vereinigung von Sparkasse und Hypothekenbank und der Anschluss eines Häuserbauverein als social-ökonomische Aufgabe unserer Zeit, Berlin 1845. Vergl. auch ähnliche Überlegungen bei EUGEN RENTSCH, Art. „Wohnungsfrage“, in: Handwörterbuch der Volkswirtschaftslehre, Leipzig 1866. wirtschaftlichen Wandels im beginnenden Industriezeitalter erkannt wurde. Wie Friedrich Engels, Bruno Hildebrand und Victor Aimé Huber stützte aber Wie Friedrich Engels, Bruno Hildebrand und belgische Großstädte, da in Deutschland abgesehen von Berlin diese Frage noch nicht zu dieser Reife gediehen war.

Im Vordergrund aller dieser Bemühungen bestand die Beschaffung von ausreichendem Wohnraum für die vom Land in die Stadt strömenden Arbeitskräfte. Während auf dem Baumarkt der Wohnungsbau in den sechziger und siebziger Jahren allmählich auf eine erste kapitalistische Grundlage gestellt und das traditionelle selbständige Maurerhandwerk in den Hintergrund gedrängt bzw. von großen Baufirmen abhängig gemacht wurde, vergunten Baugenossenschaften den unsozialen Auswüchsen des neuen großstädtischen Wohnungselends entgegenzusteuern. Diese Problematik spiegelt sich in einer Fülle von Schriften wider, die bis heute noch nicht zusammenhängend ausgewertet wurden 21 .

Eine heftige und grundsätzliche Kritik an der wachsenden Großstadt wurde in der Mitte des vorigen Jahrhunderts von Wilhelm Heinrich Riehl vorgetragen. Als Vorkämpfer einer empirischen Soziologie, Volkskunde und Kulturgeschichte sah er es ähnlich wie Justus Möser im späten 18. Jahrhundert als große Aufgabe der Zeit an, durch vergleichende Beobachtung zu "Naturgesetzen des Volkslebens" vorzudringen: Stamm, Sprache, Sitte und Siedlung (die vier großen S“) überdauerten seiner Meinung nach den Wandel aller politischen Staatsformen und sollten daher den Gegenstand wissenschaftlicher Analysen bilden. Wie die moderne Strukturgeschichte ging Riehl von langandauernden, allmählich sich vollziehenden historischen Struktur veränderungen der Völker aus, die ihre Eigenart ausmachten. Nur durch unmittelbare Anschauung, durch teilnehmende Beobachtung („Erwandern“) und naturgeschichtliche Beschreibungen von "Land und Leuten“ konnten diese hinreichend erforscht werden, nicht allein aus Akten und Urkunden oder bloßen gedanklichen Deduktionen. Riehl war keineswegs der Auffassung, eine solche „geistige Statistik der Sitten“ müsse sich auf bloße Deskription beschränken. Sein ausgesprochenes Ziel war es, von diesem empirisch erarbeiteten Material zu einem gegliederten theoretischen System vorzustoßen, mit dessen Hilfe das Zusammenspiel der Stände, Schichten und Gruppen einer

${ }^{2 !}$ Vergl. LuDolf Parisius, Bericht über die in Deutschland bestehenden Baugesellschaften und Baugenossenschaften, in: Der Arbeiterfreund. 3, 1865, S. 292-314. - W. EммісH, Betrachtungen über den Stand des Unternehmens der gemeinnützigen Baugesellschaft und über neu Vorschläge zur Abhülfe der Wohnungsnoth in Berlin, in: Zeitschrift des Central-Vereins für das Wohl der arbeitenden Klassen, 2, 1860, S. 195-201. KARL BRAMER, Uber Hâuser Uaudie Verbech Roberts mitgetheilten Nachrichten, in: Zeitschrift für das Wohl der arbeitenden Klassen, 1 , 1859, S. 150-168, - HUGO SENTTI, Die Bedeutung und der Fortschritt der Wohnungsfrage, in: Der Arbeiterfreund, 6, 1868, S. 365-400, 7, 1868, S. 822, S. 213 und S. $376 f$ f (Bericht über die in: Der Arbeiterfreund, 6, 1868, S. 365-400, 7, 1868, S. 822, S. 213 und S. $376 \mathrm{ff}$ (Bericht uber die England, in: Der Arbeiterfreund, 3, 1865, S. 233-240. - DeRs., Braunschweigische Aktiengesellschaft für den Bau von Arbeiterwohnungen nebst deren Statut, in: Der Arbeiterfreund, 8 1870, S. 122-145. - L. Parisius, Die auf dem Princip der Selbsthilfe beruhenden Baugenossenschaften, in: Der Arbeiterfreund, 3, 1865, S. 262-291. - ARWED EMminghaUs, Die Wohnungsfrage, in: Der Arbeiterfreund, 6, 1864, S. 213-253. 
Gesellschaft und damit unter anderem auch die Beziehungen zwischen Stadt und Land erfaßt werden könnten. In seiner zwischen 1854 und 1856 veröffentlichten „Naturgeschichte des Deutschen Volkes als Grundlage eine socialen Politik" war sein Blick als konservativer Gesellschaftswissenschaftle vor allem auf das in seinen Augen noch unverdorbene Leben auf dem Lande gerichtet. Die großen Städte erschienen ihm als Anhänger der alten $\mathrm{Ge}$ sellschaftsformen letztlich als ein katastrophales Verhängnis. Den Vorgang de zunehmenden Verstädterung sah er nicht überall pejorativ, da er z.B. die wohltätigen Folgen für Handel und Gewerbe infolge steigender Rationalisierung und Arbeitsteilung bemerkte. Aber diese günstigen Auswirkungen für die wirtschaftliche Produktivität galten ihm weniger als die soziokulturellen Verluste auf der anderen Seite. So kritisierte er an der entstehenden Großstad die mit ihr einhergehenden gesellschaftlichen Dekorporierungsprozesse und das neue "aufgeregte, ungesunde Seelenleben“. Mit scharfen Ausdrücken geißelte er die Formlosigkeit, Uniformität und Maßlosigkeit der „Weltstädte“, die er an den Beispielen von London und Paris selbst studiert hatte. Die moderne Großstadtkritik vom Schlage Jane Jacobs und Alexander Mitscherlichs, die sich vor allem aus sozialpsychologischen Motiven speist, aber auch die Äußerungen der neuen Grünen Bewegung unserer Tage weisen erstaunliche Parallelen zu Riehls Denkkategorien auf 22. Die großen städtischen Zusammenballungen erschienen ihm als prinzipielle Widersacher des alten organischen Lebens. Was Riehl am meisten skeptisch stimmte, waren die Massen von wandernden, aus ihrer Heimat entwurzelten, alleinstehenden jungen Menschen, die er mit einem treffenden Ausdruck die „schwebende Bevölkerung“ nannte. Der bayerische Gelehrte, der heute für eine Reihe von wissenschaftlichen Disziplinen als Stammvater gelten kann, sah das Ziel einer "Socialpolitik“ (diesen Begriff prägte er erstmals!) darin, dem entstehenden städtischen Proletariat (,Vierten Stand") rechtzeitig einen geachteten Platz in der Gesellschaft zu verschaffen, da alle Vereinzelung auf die Dauer das soziale Ganze zertrümmern würde

Ferdinand Tönnies hat diese seit Riehl vielbesprochene „Unnatur“ der modernen Großstadt auf eine höher reflektierende Ebene gebracht. In seinem Werk "Gemeinschaft und Gesellschaft" setzte er sich mit den Beziehungsmustern, Lebenskreisen und Interaktionen in der großen Stadt im Gegensatz zu den bisher dominierenden Daseinssphären Familie, Dorf, Klein- und Mittelstadt auseinander. Der Kieler Gesellschaftswissenschaftler diagnostizierte hier zwei grundsätzlich antinomische Formen menschlicher Gesellung: In den kleinen „Gemeinschaften“ lebten die Menschen trotz vielfältiger äußerer räumlicher Trennung in einer inneren emotionellen Verbundenheit, in der Metropolis dagegen trotz z.T. enger körperlicher Nähe in einer prinzipiellen Gefühlsdistanz. Auf der einen Seite sah Tönnies ein Zusammenleben auf der Grundlage enger personaler und um ihrer selbst willen bejahter Beziehungen, auf der anderen nur zweckhafte Verbindungen und dadurch bedingte zwischenmenschliche Entfremdungen. Formen der Gemeinschaft waren ihm Verwandtschaft, Nachbarschaft, Freundschaft, vor allem aber die

22 JANE JACOBS, Death and Life of American Cities, New York 1961. Deutsch: Tod und Leben amerikanischer Städte, Gütersloh 1963. - AleXANDER MitscherLICH, Die Unwirtlichkeit durch Blutsbande geprägte Familie. Hier herrschten wie in allen gesellschaftlichen Kleingebilden seiner Meinung nach Eintracht, Sitte und Religion. In den großen amorphen Sozialgebilden dominierten dagegen anonyme Unternehmen wie Aktiengesellschaften, Großstädte oder die Wirtschaftsgesellschaft schlechthin. In den makrosozialen Gesellungen wurden nur interessenspezifische Bindungen mittels loser Konventionen, Politik und öffentlicher Meinung gepflegt. Das Gemeinschaftsleben spielte sich auf der Basis großer mitmenschlicher Intensität $a b$; das Leben in den größeren Gesellschaftsformationen und vor allem in den gesichtslosen Großstädten war durch unübersichtliche Komplexität und überlokale Verflechtungen gekennzeichnet. Den kleinen organisch gewachsenen Einheiten des menschlichen Daseins traten nun erstmals in der Geschichte mechanische Artefakte der Gesellschaft gegenüber, die ihrem Wesen nach grenzenlos waren und $\mathrm{zu}$ beständiger Extension neigten 23 .

Daneben beschäftigt sich Tönnies, was bisher mangels einer Edition seines Gesamtwerks vielfach übersehen wurde, mit stadtsoziographischen Untersuchungen, insbesondere mit Selbstmord, Kriminalität und Lage der städtischen Lohnarbeiterschaft, wobei es ihm auf die Aufdeckung von Korrelationen zwischen Konjunkturverläufen, materieller Verarmung, Geschlecht und Familienverfassung auf der einen, sowie Wohnsitz in Stadt und Land auf der anderen Seite ankam. Die in der damaligen Öffentlichkeit teilweise leidenschaftlich erörterten Suicid- und Verbrechensraten in den Großstädten hatten ihm Anlaß zu diesen Forschungen gegeben. Die Daten stammten von den Statistischen Ämtern, die die Städte wie schon erwähnt eingerichtet hatten. Tönnies erblickte auch hier wieder einen Beweis für seine Grundthese, daß der Großstadtmensch ein mehr individueller, auf seine Interessen hin bedachter Typ sei, bei dem die jahrhundertealten Werte des "Gemütslebens“ im Schwinden begriffen seien, weil der schärfere Konkurrenzdruck, die Abwehr bzw. Anpassung alle seine Aufmerksamkeit erfordere. Das Verbrechen wurde von ihm ausdrücklich in diesem Zusammenhang erstmals als gesellschaftliches Phänomen klassifiziert und die Verbrechensrate der Großstädte mit den Faktoren soziale und räumliche Mobilität, Heimat- und Eigentumslosigkeit in Relation gesetzt. Merkwürdigerweise hat Tönnies, der in seinen soziologischen Theorien deutliche Anklänge an Adam Müller, Albert Schäffle, Carl Rodbertus, aber auch an Rudolph Gneist, Adolph Wagner und besonders die Sozialwissenschaftler Heinrich Ahrens, Foustel de Coulange und Henry Morgan erkennen läßt, keinerlei praktische Anwendungen aus seiner weitreichenden und bis heute diskutierten Stadtheorie gezogen oder 23 FERDINAND TönNIES, Gemeinschaft und Gesellschaft. Abhandlung des Communismus und des
Socialismus als empirische Culturformen, Leipzig 1887. Wirklich bekannt wurde das Werk erst in der zweits Reinen Soziologie, Berlin 1912 (8. Aufl. Gemeinschaft und Gesellschaft. Grundbegriffe der AlFred Bellebaum, Das soziologische System von Ferdinand Tönnies unter Vesonderer Berücksichtigung seiner soziographischen Untersuchungen, Meisenheim/Glan 1966. - RENÉ KöNIG, Die Begriffe Gemeinschaft und Gesellschaft bei Ferdinand Tönnies, in: Kölner KonIG, Die Begriffe Gemeinschaft und Gesellschaft bei Ferdinand Tönnies, in: Kölner Gruppe und die Kategorien Gemeinschaft und Gesellschaft, in: Archiv für Sozialwissenschaft und Sozialpolitik, 58, 1927, S. 338f. - Rudolf HeBerLe, Art. „Soziographie“, in: Handwörterbuch der Soziologie, Stuttgart 1931 (Nachdruck 1959). - LoUIS WIRTH, The Sociology of Ferdinand Tönnies. In: American Journal of Sociology, vol. 32 (1926-27), pp. 412ff. 
aber Vorschläge für eine Städte- oder Wohnungsreform gemacht, was in seiner Zeit nahe gelegen hätte. Seine wichtige Differenzierung zwischen dem „dörflichen Wesensverband" und dem "städtischen Zweckverband" hat offensichtlich aber in der entstehenden Gartenstadtbewegung eine größere Rolle gespielt. Der Tiefgang seiner stadtsoziologischen Thesen läßt sich auch daran ermessen, daß sich Oswald Spengler in seinem philosophischen Bestseller "Untergang des Abendlandes", der Amerikaner Lewis Mumford in seinen zahlreichen Büchern über die Stadt sowie die heutige Gemeinde- und Familiensoziologie intensiv mit ihm beschäftigt haben 24 .

Von Tönnies ausgehend wurden Familie und Gemeinde als gesellschaftliche Archetypen und als besondere lokale Gruppen von Menschen erkannt, die ihr ökonomisches und soziokulturelles Handeln nach gut abgrenzbaren Werten ausrichten und so zur sozialen Identitätsfindung des Individuums beitragen. Wichtig war dabei die Erkenntnis, daß sich diese sozialen Urphänomene, die gleichsam als Mikrokosmen einer größeren Gesellschaft angesehen werden können, in der Regel gerade nicht mit den Verwaltungsbezirken einer Stadt decken, so daß zwischen informalen und formalen Gruppenbeziehungen in der städtischen Gemeinde unterschieden werden muß. Für die empirische Nachbarschaftssoziologie wie auch die Umweltpsychologie als neueste Zweige der Urbanistik sind solche Einsichten von Nutzen geblieben 25

Wissenschaftsgeschichtlich noch folgenreicher für die Entwicklung der modernen Großstadtforschung wurde der aus Berlin stammende Philosoph und Gesellschaftswissenschaftler Georg Simmel. Auf Einladung der an Städteund Wohnungsreform besonders interessierten Gehe-Stiftung hielt er anläßlich der Städteausstellung von 1903 in Dresden einen Vortrag mit dem Titel „Die Großstädte und das Geistesleben", in dem er die Stadt vor allem als einen Ort der modernen Geldwirtschaft und der sich in ihrem Gefolge ausbreitenden Prozesse der Arbeitsteilung und Rationalisierung begriff 26 . Ähnlich wie Tönnies beschäftigte er sich mit der psychischen Mentalität des modernen Groß-

${ }^{24}$ Lewis Mumford, City Development, New York 1945. Deutsch: Megapolis - Gesicht und Seele der Großstadt, Wiesbaden 1951. - Ders.; The Culture of Cities, New York 1938, (2. Aufl. London 1946). - The City in History, New York 1961. Deutsch: Die Stadt, Köln 1963. - ReN KöNIG, Art. "Gemeinde“, in: Fischer-Lexikon Soziologie, Frankfurt/M. 1958, S. 73-83. HeLMUT SCHELSKY, Wandlungen in der deutschen Familie, Stuttgart, 1964. - RENÉ KöNIG, Grundformen der Gesellschaft: Die Gemeinde, Hamburg 1958. - Ders., Soziologie de Gemeinde, in: Kölner Zeitschrift für Soziologie und Sozialpsychologie, 1, 1963. Vergl. HERMANN KORTE, Soziologie der Stadt, 2. Aufl., Muñnhen 1974. - Elisabeth PFeIL, Großstadtforschung. 2. neubearb. Aufl.,
Stadtplaner, Frankfurt a.M. 1968 .

25 HeLMUT KLAGs, Der Na. 1ie nachbarschaftliche Wirklichkeit in der

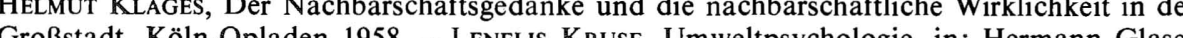
.

26 GEORG SIMMEL, Die Großstude und das Geistesleben, in: Die Großstadt. Vorträge und Aufsatze zur Städteausstellung, (Jahrbuch der Gehe-Stiftung, 9) Dresden 1903, S. 185ff. Wunst, Religion und Gesellschaft, hrsg. von Michael Landmann und Margret Susman Stuttgart 1957. - Vergl. RuDOLF TARTLER, G. Simmels Beitrag zur Integrations- und Konflikttheorie der Gesellschaft, in: Jahrbuch für Sozialwissenschaften, 16, 1965, S. 1ff. - KURT H. Wolff, The Sociology of G. Simmel, Glencoe, Ill., 1950. - PeTer E. Schnabel, Die soziologische Grundkonzeption Georg Simmels, Stuttgart 1974. - Georg Simmel, Philosophie des Geldes, München-Leipzig 1900 (6. Aufl. 1958) tädters, ohne aber der damals vorherrschenden Großstadtfeindschaft und Agrartümelei zu verfallen. Nüchtern versuchte er, die Auswirkungen dieser Agrartu Lebenssphäre auf das menschliche Dasein insgesamt abzuwägen. De neuen Lebenssphäre auf das menschliche Dasein insgesamt abzuwägen. Der
Großstädter, so behauptete der deutsch-jüdische Gelehrte, lege gleichsam als Schutzfunktion gegenüber seinen zahlreichen Mitmenschen eine neue, von einer gewissen Blasiertheit nicht freie Reserve an den Tag, die Landmenschen nicht kennen. Simmel erblickte in dieser versachlichten und entemotionalisierten Haltung des Großstädters einen gewissen Selbstschutz vor zu vielen Kontakten und einer psychischen Atomisierung. Die neue soziale Distanzierung ermöglichte den metropolitanen Bewohnern die Bewahrung ihre individuellen Unabhängigkeit und schuf damit neue Chancen zur geistigen Entwicklung. Emanzipation und Arbeitsteilung. Simmel, der trotz seiner sonst sehr formal gehaltenen Gesellschaftslehre die historische Methode und empirische Verfahren zur Überprüfung genereller soziologischer Modelle und Hypothesen prinzipiell befürwortete und vor allem an den Vergesellschaftungsformen interessiert war, die in relativer Konstanz einen sozialen Wande auf "lange Dauer" bewirkten, sah in dem Urbanisierungsprozeß insgesamt einen positiv zu bewertenden Vorgang der Vergemeinschaftung und keinen dekadenten Kulturverfall wie Wilhelm Heinrich Riehl ${ }^{27}$. Die Großstadt eröffnete bisher nicht gekannte Möglichkeiten der Individuation für den Men schen, bescherte freilich vice versa auch neue soziale Zwänge und Abhängigkeiten.

In den USA ist die Entstehung der „Urban Sociology“, insbesondere der berühmten Chicagoer Schule, am Anfang durch Simmel und seine optimistische Sicht stark beeinflußt worden. Die Wertschätzung des Berline Urbanisierungsforschers geht aus den ersten amerikanischen Veröffentlichungen und Übersetzungen Simmels hervor. So setzte Robert Ezra Park schon in seinem 1916 erschienenen Aufsatz „The City-Suggestion for the Investigation of Human Behavior" in betonter Anlehnung an Simmels klassische Abhandlung von 1903 und die Übersetzungen durch seinen Kollegen Albion W.

${ }^{27}$ Die Bewertung der Großstadt korrespondiert mit Simmels Auffassung vom Wesen der Geldwirtschaft und der Modernisierung: Die Akzeptierung des Geldes als Zahlungmittel hat die relativ geschlossene jahrtausendealte Naturalwirtschaft aufgelost, Kommunikationen und Handel zwischen distanziertesten Partnern ermöglicht und vor allem die halbsklavische Abhängigkeit des Individuums bei der naturalwirtschaftlichen Entlohnung vermindert. Die neue Geldwirtschft des kapitalistischen Systems erfordert nur noch partielle Unterwerfungen der Persönlichkeit, hat freilich dafür andere sublimere Formen menschlicher Unfreiheit erzeugt, weil sie die Menschen nun zwingt, sich im Konkurrenzkampf zu profilieren. Die mit der modernen Geldwirtschaft verbundene Versachlichung sozialer Herrschaftsbeziehunge und zwischenmenschliche Distanzierung in den unmittelbaren Lebensbereichen hat Simmel ganz olfenbar zu seiner These uber die geistig-sechsche Physiognustrialisierung und Urbnisierung von en worgeldwirtschaftlichen Kulturen und kann prinzipiell nun alles frei kaufen und verkaufen. Zugleich ist er aber unf̈̈hi die sich neu ergebenden Freiheitsräume zum Zweck der Selbstverwirklichung voll zu nutzen und begibt sich in neue, selbst geschaffene Abhängigkeiten. Änlich wie Max Weber, der durch Simmels "Philosophie des Geldes" seiner berühmten Studie „Die protestantische Ethik und der Geist des Kapitalismus“ mitseiner berühmten Studie „Die protestantische Ethik und der Geist des Kapitalismus mitzu rechtfertigende idealistische Gesamttheorie widerlegen. Plumpe Apologien bestehender Verhältnisse liegen ihm fern. Die sozialen Folgen der ökonomischen Strukturveränderungen sind ihm wie Marx unerhört wichtig für die gesellschaftliche Bewußtseinsbildung; alle zwangsläufigen Einseitigkeiten quasi-gesetzmäßiger Art werden aber von ihm strikt abgelehnt. 
Small einen ersten Schwerpunkt für die Urbanisierungsforschung in den Vereinigten Staaten, deren eigene Ergebnisse neun Jahre später unter Mitarbeit von W. E. Burgess, R. D. MacKenzie und L. Wirth in dem berühmten Sammelwerk "The City" 1925 vorgelegt wurden 28 . Wenngleich der Name Simmel bei den nachfolgenden Urbanisierungsforschern kaum noch erwähnt wurde, so lassen doch viele Problemstellungen und die theoretischen $\mathrm{Be}$ zugsrahmen der überwiegend empirisch angelegten soziologischen Untersuchungen den Simmelschen Einfluß noch gut erkennen 29. Nach dem 2. Weltkrieg besann man sich im Rahmen einer Simmel-Renaissance dieses wichtigen wissenschaftlichen Erbes; es wurden nicht nur die wichtigsten Schriften dieses deutschen Gelehrten neu und gesammelt herausgegeben, sondern auch ihm ein "Buch des Dankes" gewidmet 30 .

Einen gänzlich anderen Ansatz zur theoretischen Erfassung der Stadt und Verstädterung lieferte der Nationalökonom Werner Sombart. Sein Blick ruhte primär auf den ökonomischen Funktionen der Stadt. Im Rahmen seiner bekannten Stufentheorie über die Entwicklung des "modernen Kapitalismus" stellte er folgende Fragen 31 :

- Woher kamen die Menschen für die Städte?

- Was veranlaßte sie zum Zusammenschluß einer städtischen Siedlung?

- Wie wurde es möglich, daß sich diese urbanen Zusammenballungen der "natürlichen" agrarischen Lebensweise entfremden konnten?

In seiner um 1900 konzipierten Theorie der Städtebildung hat Sombart u.a. die wissenschaftliche Vermutung geäußert, die Größe einer städtischen Siedlung habe zu Beginn von der Höhe der landwirtschaftlichen Überschüsse des Umlandes, bzw. genauer gesagt, von dessen Bodenfruchtbarkeit, dem Stand der landwirtschaftlichen Technik und der Lage abgehangen. Der Einfluss der klassisch-liberalen Wertlehre sowie der darauf basierenden Standorttheorien läßt sich hier erkennen. Als einer der begrifflich anregendsten Gelehrten seiner Zeit unterschied Sombart ferner zwischen den "primären Städtegründern" (Landes- und Grundherren bzw. Staat) und „sekundären Städtefüllern“, die von den Einkünften der ersten Gruppe lebten. Daran schlossen sich dann wiederum „tertiäre Städtefüller", die sich wiederum auf die Einnahmen der zweiten Gruppe stützten. Das in der Folgezeit viel diskutierte "Gesetz vom doppelten Stellenwert" geht auf diese Sombartsche Städtetheorie zurück 32.

28 Robert Ezra Park et. Al., The City, Chicago 1925. - Vergl. Ernest Burgess (ed.), The Urban Community, Chicago 1926

29 Vergl. unter anderem N. Anderson, The Hobo (1921). - E. Thrasher, The Gang (1927). Le M. Lind Middle-Town in Tra Cities (1937). - EDward H. SHIS, The Metoplis and Mental Life (1937). - Hans $H$. GERTH/C. WRIGHT MILLS, The Metropolis and Mental Life, Ann Arbor. Mich., 1949.

30 KuRT GAssen/Mies 1958. GASSEN/Michael LANDMANN (Hrsg.), Das Buch des Dankes an Georg Simmel, Berlin 1958. - LEWIS H. COSER, Georg Simmel, Englewood Cliffs, N.J., 1965. - Vergl. ERIC LAMPARD,
American Historians and the Study of Urbanisation, in: American Historical Review, 67, 1961. - Philop HAuSER/Leo F. SCHNORE (eds.), The Study of Urban History, New York 1965.

31 WerNer Sombart, Der Begriff der Stadt und das Wesen der Städtebildung, in: Archiv für Sozialwissenschaft und Sozialpolitik N.F., 25, 1907, S. 1-9. Später aufgenommen in: DeRS., Der moderne Kapitalismus, Bd. 1, 1. Halbbd., Berlin 1928, S. $124 \mathrm{ff}$ (3. Aufl., Bd. 3, 1. Halbbd., Berlin 1955, S. 399ff. Separater Neudruck Berlin 1979).

32 Sombart hat das ubiquitäre Schema des „Städtefullers an folgendem Beispiel demonstriert. Wenn in einem Restaurant ein Kellner ein Glas Bier trinkt, so lebt davon der Wirt. Von diesem
Sombart hat andere Einflüsse bei einer Städtegründung keineswegs überehen, war aber der Meinung, daß eine theoretische Erfassung des vielschichtigen Phänomens der Stadt nur durch solche raumwirtschaftliche Abstraktionen möglich sei. Er kritisierte, die Historikerzunft hätte sich fast ausschließlich mit der rechtlichen Entstehung der Stadtverfassung beschäftigt und dabei die Erscheinungen des realen wirtschaftlichen und sozialen Lebens vernachlässigt oder übersehen. Nicht den städtischen Verfassungsnormen, sondern der städtischen Verfassungswirklichkeit müsse man verstärkt nachspüren. Der Gang der bisherigen stadtgeschichtlichen Forschung sei zwar verständlich, weil die Masse der überlieferten Quellen zur Stadtgeschichte Rechtsvorgänge widerspiegele. Zur Geschichte einer Stadt und der Verstädterung gehörten aber auch die Auswertung von Chroniken, Bevölkerungs- und Vermögensstatistiken, die Geschichte von Kirchen und Klöstern sowie vor allem die Anwendung kartographischer Methoden. Erstmals wurde damit auch die Forderung nach einer sozial- und wirtschaftsräumlichen Stadtgeschichte als Ergänzung der bisherigen rechts- und verfassungsgeschichtlichen Sicht erhoben.

Die von den Rechts- und Verfassungshistorikern aufgestellte Marktrechtstheorie verwarf Sombart: Seiner Meinung nach sind die Städte nicht entstanden, weil Märkte abgehalten wurden, sondern es wurden erst dort regelmäßig Waren getauscht, wo bereits Städte bestanden bzw. im Entstehen begriffen waren. Das Marktrecht ist seiner Ansicht nach für die weitere Ausbildung der Stadtverfassung zwar wichtig geworden, doch war es für die Stadtgründung im ökonomischen Sinne nicht entscheidend. Sombart bezweifelte im Anschluß daran, ob es im europäischen Mittelalter überhaupt Gebilde gegeben habe, die ökonomisch den Begriff "Stadt“ verdienen. Nach Sombarts Ansicht sind die Städte in einem über die Jahrhunderte sich erstreckenden Umbildungsprozeß allmählich aus Dörfern und Siedlungen emporgewachsen und nicht durch einen plötzlichen Gründungsakt ins Leben getreten. Unter Verweis auf die Untersuchungen von Maurer, Below, Lamprecht und Bücher (dessen Buch „Die Bevölkerung Frankfurt am Mains im 14. und 15. Jahrhundert" als bestes Werk der deutschen Stadtgeschichte bezeichnet wurde!) stellte er die These auf, auch die größeren Städte des Hoch- und Spätmittelalters seien in Wahrheit nur kleine „Ackerbürgerstädte“ gewesen, weil die Mehrheit ihrer Bewohner allein oder doch überwiegend noch von der Landwirtschaft gelebt habe ${ }^{33}$. In ausführlichen Darlegungen hat dann Som-

bezieht der Bierbrauer sein Einkommen. Der Kellner bezahlt mit dem Trinkgeld, das er von einem Gast bekommen hat, der Arzt ist, der seinerseits es von einem Patienten kassiert hat. Dieser hat sein Geld wiederum als Schauspieler aus einem Theaterfonds bekommen, in dem z.B. ein Professor sein Geld für Eintrittskarten bezahlt hat. Jener bezieht wiederum sein Gehalt
vom Staat. Alle diese erwähnten Berufsgruppen sind nach Sombart Städtefüller"“, der Staat vom Staat. Alle diese erwähnten Berufsgruppen sind nach Sombart „Städtefüller“, der Staat
(bzw. seine Vorläufer) allein der Städtegründer. Dieses Schema wird dann an historischen (bzw. seine Vorläufer) allein der Städtegründer. Dieses Schema wird dann an historischen bestimmten Standort das Wesen einer industriellen Gesellsch ft Areitsvermogens an einem ein Zun stand das Wrest Kreislauf von den primären Arbeitseinkommen über alle privaten und öfentlichen Haushalte Kreislauf von den primären Arbeitseinkommen über alle privaten und offentire
von jeder primären Stelle eine zweite in den Folgeleistungen mitgetragen wird.

von jeder primären Stelle eine zweite in den Folgeleistungen mitgetragen wird. Sombart hielt auch die von den Historikern vertretene Ansicht für grotesk, im Rahmen der
deutschen Ostsiedlung sei ,planmäßig“ ein Netz von Gründungs- und Kolonisationsstädten deutschen Ostsiedlung sei „planmäßig“ ein Netz von Gründungs- und Kolonisationsstädten
entstanden. Seiner Meinung nach habe es sich vielmehr zunächst um Militärkolonien gehandelt wie sie auch die Römer zuerst anlegten. Die Bewohner lebten hauptsächlich vom Ackerbau und nicht vom Handel. 
bart anhand der historischen Quellen einzelne Gruppen der "Städtebildner“ und "Städtefüller“ beschrieben. Da die ersten Städte seiner Meinung nach

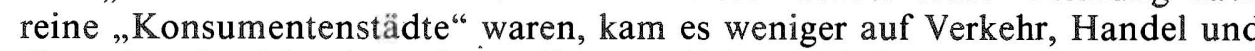
Gewerbe als vielmehr auf Bevölkerungsdichte und das agrarische Mehrprodukt des Umlandes an, das die Stadt verzehrte ${ }^{34}$. Für das Städtewachstum se daher im Spätmittelalter bzw. der frühen Neuzeit die in den Städten konsumierten Grundrenten und nicht das dort akkumulierte Handelskapita entscheidend gewesen. Sombarts Thesen dienten in erster Linie dazu, die Entstehungen der modernen industriellen Wirtschaft und damit auch das Wesen der Großstadt des 19. Jahrhunderts durch Vergleiche mit den davorliegenden Jahrhunderten besser herauszuarbeiten. Die Fachhistoriker haben sich mit dem hier gezeichneten Bild des frühen Städtewesens vielfach nicht einverstanden erklärt und vor allem Sombarts Theorie über die Rolle der Grundrenten für die Städtegründung durch Vorlage von Quellen zu erschüttern versucht 35 .

Der Streit zwischen dem Nationalökonomen Werner Sombart und den Vertretern der Geschichtswissenschaft um die Jahrhundertwende ist inzwischen von der Forschung überholt worden und interessiert heute nur noch vom wissenschaftsgeschichtlichen Standpunkt. Die Historiker hatten sicherlich die größere Quellenkenntnis und in vielen Einzelheiten recht, so daß Sombar seine Stadtheorie erheblich revidierte. Dabei muß allerdings festgehalten werden, daß die Fachhistoriker im Gegensatz zu Sombart auch in der Folgezeit keinen Beitrag zur Begriffsbestimmung der Stadt geleistet haben. Solche generalisierenden Betrachtungen blieben ihnen weiterhin fern.

Eine Weiterentwicklung der Stadttheorie geschah durch Max Weber. Wie Sombart interessierte er sich in erster Linie für die wirtschaftlichen Funktionen der Städte, insbesondere für die Entstehung der ersten Marktsiedlungen, die Typen von Konsumenten- und Produzentenstädten und ihre Beziehungen zu der umgebenden Landwirtschaft, die Herausbildung der einzelnen Stufen der Stadtwirtschaft, dann aber auch für die Genesis des politisch-administrativen Stadtbegriffs ${ }^{36}$. Seine Darlegungen beschäftigten sich ferner mit der Verschmelzung der militärischen Festungen und Garnisonen mit den Märkten

${ }^{34}$ Sombart ist hier offensichtlich Büchers bekannter Stufentheorie gefolgt, die eine Abfolge von Stadt-, Territorial- und Volkswirtschaft sah. Heute wird bekanntlich nicht mehr angenommen die "Stadtwirtschaft" habe eine besondere Periode dargestellt. Neben der sich aus dem Umland mit Lebensmitteln versorgenden und dieses mit Gewerbeprodukten wiederum beliefernden lokalen Marktstadt gab es frühzeitig überwiegende Fernhandels-, Gewerbe-, Hafen- und Bergstädte. Später kamen dann die speziellen Residenz-, Verwaltungs-, Universitäts-, Garnisons- bzw. Festungsstädte hinzu. Neben der nur auf das engere Umland angewiesenen Stadwirtschaft gab es frühzeitig auch eine Territoig so

I. Z.B. JAKOB STRIEDER, Zur Genesis des Kapitalismus. Forschungen zur Entstehung großer Tittelalters und der frühen Neuzeit, Leipzig 1903.

MAX WEBER, Die Stadt, in: Archiv für Sozialwissenschaft und Sozialpolitik N.F., 47, 1921, S. $621 \mathrm{ff}$. - Vergl. DeRs., Wirtschaft und Gesellschaft. Grundriß der verstehenden Soziologie, hrsg. Herrschaft (Typologie der Städte). Webers Abhandlungen über die Stadt sind auch gesondert ins Englische übersetzt worden: MAX WEBER, The City. Translated and edited by Don Martinsdale and Gertrud Neuwirth, New York 1958. sowie dem sozialen Verbandscharakter der ersten Stadtgemeinden und der städtischen Qualifikationen des neuen "Bürgers". Schließlich beschrieb er antike und mittelalterliche Stadtverfassungen, denen er mit großer Meisterschaft verschiedene Stadttypen des alten Orients, Chinas und Indiens gegenüberstellte. Auf hoher Abstraktionsstufe stehend interessierte sich Max Weber für die Entstehung des „okzidentalen Kapitalismus“, in dem die Städte als besondere Formen rationalisierter Herrschaft eine große Rolle spielten. Auf die engeren Probleme der Urbanisierung im 19. Jahrhundert ist er bei seinen über weite Zeiten und Räume angelegten Betrachtungen aber nicht eingegangen. Neben diesen Versuchen, zu einem theoretischen Rahmenkonzept für die Stadt und die Stadtentwicklung zu kommen, gab es eine Fülle von Literatur, die sich weiterhin mit den Ursachen und Folgen der Wohnungsnot im 19. Jahrhundert beschäftigte. Im Vordergrund standen seit den siebziger Jahren konkrete Bestandsaufnahmen und Vorschläge zur gesetzlichen Abhilfe der festgestellten Übelstände. Einen Markstein in dieser engeren Urbanisierungsliteratur bildet ohne Zweifel die von dem Berliner Statistiker Ernst Engel 1873 veröffentlichte Schrift „Die moderne Wohnungsnoth“, die Anlaß zur Formulierung des Engel-Schwabeschen Gesetzes über die Relation der Wohnungskosten zu den allgemeinen Lebenshaltungskosten wurde. Nicht weniger beeindruckte die Zeitgenossen die von Emil Sax erstmals vorgelegten Unterscheidungskriterien der ländlichen und städtischen Wohnungsnot und der daraus entwickelte Katalog schichttypischen Wohnens ${ }^{37}$. Der Verein für Socialpolitik veranstaltete ebenfalls in diesen Jahren eine erste große Enquete in Deutschland und veröffentlichte sie 1886 unter dem Titel „Die Wohnungsnot der ärmeren Klassen in deutschen Großstädten“ 38 . Erstmals wurde anhand von Gutachten und Statistiken genauer Aufschluß über das Verhältnis der Bevölkerung zur bebauten Fläche, zu den Grundstücken, Gebäuden und Wohnungen sowie über die schichtspezifische Verteilung der Haushalte und Mietausgaben gegeben. Diese erste große empirische Bestandsaufnahme über die Folgen der Urbanisierung in Deutschland verstärkte die Ansicht, daß den Kommunen hier ganz neue Aufgaben gestellt seien und führte zur Gründung eines „Vereins Reichswohnungsgesetz“ 1898 (später Verein für Wohnungsreform). Nach Ansicht seiner Mitglieder sollten vom Gesetzgeber folgende Probleme geregelt werden ${ }^{39}$ :

- Einführung einer allgemeinen Wohnungsinspektion

- Schaffung einer staatlichen Genehmigungs- und Revisionsinstanz für Bauordnungen und Bebauungspläne

- Heranziehung öffentlicher Kredite zur Förderung des Kleinwohnungsbaus

- Reform des Enteignungrechts und Erneuerung der Bestimmungen über den

37 ERNST ENGEL, Die moderne Wohnungsnoth, Signatur, Ursachen und Abhülfe, Leipzig 1873. Vergl. Ders, UUber die Wohnungnoth, in, Vignatur, Unsachen und Abrelfe, Leipzig 1873. Besprechung der socialen Frage, Leipzig 1872. - EMIL SAX, Die Wohnungszustände der arbeitenden Klassen und ihre Reform, Wien 1869. - DERS, Referat zur Wohnungsnot. Berichte des Volkswirtschaftlichen Kongresses, Leipzig 1873, S. 104ff.

38 Die Wohnungsnot der ärmeren Klassen in deutschen Großstädten und Vorschläge zu deren Abhülfe. Gutachten und Berichte, hrsg. vom Verein für Socialpolitik, in: Schriften des Vereins für Socialpolitik, 30-31, Leipzig 1886.

${ }^{39}$ Vergl. Deutscher Verein für Wohnungsreform (Hrsg.), 30 Jahre Wohnungsreform 1898-1928, Berlin 1928. 
Grundbesitz von Gemeinden

- Reform des Mietrechts, des Mietprozesses und der Zwangsvollstreckung

- Entwicklung und Verbilligung des städtișchen Nahverkehrs

- Errichtung eines Reichswohnungsamtes als Abteilung des Reichsamts des Innern.

Die weitreichenden und für die damalige Zeit revolutionären Forderungen wurden in zahlreichen Schriften heftig diskutiert und von einzelnen Vereinsmitgliedern verteidigend differenziert und konkretisiert. Die von dem Verein herausgegebene Reihe „Die Wohnungsfrage und das Reich", die seit $1900 \mathrm{zu}$ erscheinen begann, setzte diese Erörterungen in geschlossener Form fort.

Die Behandlung der Wohnungsfrage geriet durch die Bestrebungen des Vereins für Wohnungsreform, in dem auch führende Sozialdemokraten mitwirkten, immer mehr in eine Auseinandersetzung über Bodenmonopol und Bodenspekulation. Neben allgemeine theoretische Erwägungen, wie sie Friedrich Engels schon 1873 in seiner Schrift "Zur Wohnungsfrage" angestellt hatte traten nun Untersuchungen über die konkreten Ursachen. Der Verein für Socialpolitik sah sich veranlaßt, auch hierzu eigene Untersuchungen vorzulegen, die die erste Bestandsaufnahme von 1886 wesentlich ergänzten 40 .

War die ältere Bodenreformbewegung mit ihren Hauptvertretern Adolph Damaschke und Franz Oppenheimer noch von einem allgemeinen Bodenmonopol ausgegangen und damit innerhalb der klassischen Werttheorie verblieben, so konzentrierte sich nach der Jahrhundertwende die Städte- und Wohnungsreformbewegung auf spezifische Untersuchungen des städtischen Baubodenmonopols, das in unterschiedlicher Weise für die allseits beklagten Mißstände verantwortlich gemacht wurde. Karl von Mangoldt, Rudolf Eberstadt und Carl Johannes Fuchs traten hier besonders mit ihren Veröffentlichungen hervor ${ }^{41}$. Die Wohnungsfrage wurde nun in ihrer gesamten wirt-

40 Neue Untersuchungen über die Wohnungsfrage in Deutschland und im Ausland. In: Schriften des Vereins für Socialpolitik Bd. 94-97, München-Leipzig 1901. - Vergl. Verhandlungen des Vereins für Socialpolitik über die Wohnungsfrage und Handelspolitik. In: Schriften des Vereins für Socialpolitik Bd. 98, Leipzig 1902, S. 15-118.

${ }^{41}$ ADOLPH DaMASChKE, Die Bodenreform. Grundsätzliches und Geschichtliches zur Erkenntnis und Überwindung der sozialen Not, Berlin 1902 (13. Aufl. Jena 1916). - Franz OpPENHEIMER, Großgrundeigentum und soziale Frage, Berlin 1898. - DeRs., Wohnungsart und Wohnungsreform in England, Leipzig 1900. - KARL VON MANGOLDT, Die städtische Bodenfrage, Gottingen 1907.- RUDOLF EBERSTADT, Städtische Bodenfrage, Berlin 1894. - DERS., Rheinische 1903. - DeRs., Besprechung des Entwurfs eines Gesetzes zur Verbesserung der Wohnungsverhältnisse in Preußen, in: Archiv für Sozialwissenschaft und Sozialpolitik N.F Wohnung173-202. - Ders., Die Spekulation im neuzeitlichen Städnd Sozialpolitik N.F., 1, 1904, S. städtische Bodenrente und Bodenspekulation, in: Archiv für Sozialpolitik N.F., 22-23, 1906, S. 631-663. - DERS., Das Wohnungswesen, Jena 1922. - DERS. Art. Wohnungsfrag““ "Wohnungswesen“, in: Handwörterbuch der Staatswissenschaften, Jena 1929, S. 1098-1160. "Vergl. C. STEINBRǗcK, Die Entwicklung der Preise des städtischen und ländichen Immobilienbesitzes zu Halle (Saale) und im Saalekreis, Jena 1901. - H. PETER, Wert und Preis unbebauter Liegenschaften in der modernen Großstadt. Dargestellt aufgrund der Verkäufe unbebauter Liegenschaften in Mannheim 1895-1906, Karlsruhe 1910. - W. STREHLOW, Die Boden- und Wohnungsfrage des rheinisch-westfälischen Industriebezirkes, Essen 1911. - H. CONERT, Die sächsischen Terraingesellschaften und ihr Einfluß auf die Stadterweiterung, Leipzig 1911. - Alfred Baron, Die Haus- und Grundbesitzer Preußens einst und jetzt, Jena 1911 schaftlichen, sozialen und kommunalpolitischen, aber auch in ihren rechtlichen und technischen Aspekten abgehandelt und die komplexen Zusammenhänge zwischen Städtereform und Sozialer Frage bloßgelegt. Wie sehr dieser Fortschritt in der Urbanisierungsforschung auf Anstößen beruhte, den der Verein für Socialpolitik gegeben hatte, läßt sich daraus ersehen, daß der führende Kopf unter den deutschen Wohnungsreformern, der aus einer alten jüdischen Familie in Frankfurt am Main stammende Privatdozent Rudolf Eberstadt ein Schüler Gustav Schmollers war. Dieser gehörte bekanntlich zu den maßgeblichen Gründern des Vereins für Socialpolitik und hatte selbst eine Schrift mit dem Titel "Mahnruf in der Wohnungsfrage" verfaßt. Zwischen Rudolf Eberstadt und Johannes Fuchs auf der einen und den Nationalökonomen Voigt, Geldner und Philippovich auf der anderen Seite entspann sich auf der Tagung des Vereins für Socialpolitik 1902 beim Thema städtische Bodenreform eine Kontroverse, die anschließend in verschiedenen Schriften weiter ausgefochten wurde. Dabei ging es um die Frage, ob der Mietpreis den Bodenwert bestimme oder umgekehrt. Voigt und Geldner vertraten hier die These, großstädtische Wohnungsnot sei vor allem aus Mangel an geeigneten Bauunternehmungen entstanden und suchten die Wohnungsnot durch Vermehrung des Wohnungsangebots sowie größere Freiheit der kommunalen Bauordnungen marktwirtschaftlich zu lösen. Philippovich meinte demgegenüber, nicht der Bau von Kleinwohnungen, sondern die Hebung des Einkommens bei den unteren Bevölkerungsschichten sei vorrangig zu betreiben ${ }^{42}$ Das an diese Debatte sich anschließende Schrifttum hat sich immer wieder mit diesen beiden gegensätzlichen Positionen auseinandergesetzt und eine Fülle neuer Argumente und Einsichten herbeigetragen. Die Schriften von Adolph Wagner, Lujo Brentano, Adolf Weber, Ludwig Pohle, Emil Lederer und Heinrich Herkner sind in diesem Zusammenhang zu nennen ${ }^{43}$. Fast alle diese Autoren gehörten dem Verein für Socialpolitik bzw. dem „Verein Reichswohnungsgesetz" an.

Daß diese Wissenschaftler nicht nur theoretisierten, beweist ihr Eintreten für die von England ausgehende Gartenstadtbewegung. In Anlehnung an Vorschläge von Ebenezer Howard war dort Ende des 19. Jahrhunderts eine erste "Garden City Association" entstanden, die eine bewußte städtische Dezentralisierung und planmäßige Organisation von Städtegründungen sowie eine

${ }^{2}$ Eugen von Philippovich, Correferat, in: Verhandlungen des Vereins für Socialpolitik über die Wohnungfrage und die Handelspolitik, in: Schriften des Vereins für Socialpolitik, 98, 1902, S. 43-96. - ANDREAS VOIGT/PAUL GELDNER, Kleinhaus und Mietskaserne, 1905. - Ders., Zum 43-96. - ANDREAS VOIGT/PAUL GELDNER, Kleinhaus
Streit um Kleinhaus und Mietskaserne, Dresden 1907. ${ }^{43}$ ADOLPH WAGNER, Die Abschaffung des privaten Grundeigentums, Leipzig 1870 - DeRs., Wohnungsfrage in Berlin und seinen Vororten, Berlin 1901. - LuJO BRENTANO, Wohnungszustände in München, München 1903. - ADOLF WEBER, Über Bodenrente und Bodenzustande in Müchen, Munchen 1903. - ADOLF WEBER, Uber Bodenrente und Boden-
spekulation in der modernen Stadt, Leipzig 1904. - DERS., Die Großstadt und ihre sozialen spekulation in der modernen Stadt, Leipzig 1904. - DeRS., Die Großstadt und ihre sozialen burg/Br. 1863-1902 mit einem Vorwort von Carl Johannes Fuchs, Karlsruhe 1905. - LuDwiG POHLE, Der Wohnungsmarkt unter der Herrschaft der privaten Bauspekulation, in: Zeitschrift für Sozialwissenschaft, 7, 1904. - Ders., Die neuere Entwicklung der Wohnungsfrage, 2 Bde. Leipzig 1910. - EmIL LeDERER, Bodenspekulation und Wohnungsfrage, in: Archiv für Sozialwissenschaft und Sozialpolitik N.F., 25, 1907, S. 613-648. - HEINRICH HERKNER, Wohnungsfrage und Bebauungsplan, Berlin 1908. - Ders., Die Arbeiterfrage, 5. erw. Aufl., Berlin 1908 , S. 380-394. 
humane Verbindung von industriellen Arbeitsstätten und Siedeln im Grünen zum Ziel hatte. In den zu gründenden neuen Städten"sollte an nicht mehr als 30.000 Einwohner das Gemeindeland pachtweise zu Wohn-, Industrie- und Landwirtschaftszwecken abgegeben werden, wobei die Stadt selbst aber nicht mehr als ein Sechstel der vorgesehenen Bodenfläche einnehmen durfte. Deutsche Städtereformer, die schon vor Howards Auftreten unabhängig davon ähnliche Gedanken geäußert hatten, gründeten 1902 eine ,Deutsche Gar-

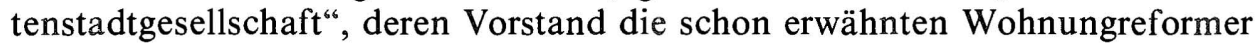
Mangoldt, Eberstadt und Fuchs angehörten. Die von der Gesellschaft herausgegebenen Schriften suchten entsprechende gesetzgeberische Maßnahmen zu initiieren und die Forderungen der englischen Schwesterbewegung den anderen deutschen Verhältnissen anzupassen 44. Da es im Deutschen Reich andere Agrarverfassungen und Städteordnungen als in Großbritanien gab, kam es nur zur Gründung von Gartendörfern bzw. Gartenvorstädten, also betont ländlichen Wohnsiedlungen innerhalb von industriellen Großstädten. Der Grundgedanke blieb faszinierend: durch Ausweichen auf billiges, von steigender städtischer Grundrente nicht erfaßtes Bauland die Hauptursachen der großstädtischen Wohnungsnot auszuschalten und eine partielle Rückkehr zur "Natur" einzuleiten. Die Vorherrschaft der Mietskaserne sollte an der Wurzel bekämpft werden. Der Sozialdemokrat Paul Kampffmeyer als Vorsitzender der Deutschen Gartenstadtgesellschaft wies auf die Grenzen seiner "grünen“" Bewegung hin: Sie konnte nicht den Anspruch erheben, die gesamte Bodenfrage oder gar die gesamte Problematik der Urbanisierung, geschweige denn der Sozialen Frage zu lösen. Es waren und blieben beschränkte Experimente mit bewußt pragmatischer Zielsetzung. Gegner der Gartenstädte wie der Kommunalpolitiker Hugo Lindemann warfen ihren Verfechtern utopischen Dilettantismus vor, weil sie die städtische Grundrentenpreisentwicklung nicht im geringsten allgemein zu beeinflussen vermochten.

In der Zeit zwischen den beiden Weltkriegen ist auf den eingeschlagenen Wegen der Urbanisierungsforschung nur teilweise weiter gearbeitet worden.

${ }^{44}$ Ebenezer Howard, Tomorrow, London 1898, 2. Aufl. unter dem Titel: Garden Cities of Tomorrow, London 1902. Deutsch: Gartenstadt in Sicht (1908). Zitiert nach: Das Buch und seine Geschichte, hrsg. von Julius Posener, Berlin-Freiburg-Wien 1968. - H. LINDEManN, Die Gartenstadtbewegung, in: Sozialistische Monatshefte, 7, 1905, S. 603-608. - PAuL KAMPFFMEYER, Zur Gartenstadtbewegung, in: Ebd., 11, 1905, S. 958-966. - Schriften der Deutschen Gartenstadtgesellschaft, Dresden 1907. - Deutsche Gartenstadtgesellschaft, (Hrsg.), Flugschriften „Vermählung von Stadt und Land“", „Abkehr von der Großstadt", „Genossenschaften und Genossenschaftsstädte“, „Der Zug der Industrie auf das Land“, "Gartenstadt und asthetische Kultur , "Thesen zur Wohnungs- und Siedlungsfrage" usw., Die deutsche Gargart 1900 - E ROTH, Litsäze und Refote Land mit anschließender Diskusion in: Deutsche Vierteljahresschrift für öffentliche Gesundheitspflege, 1, 1903. - Die hiermit eng zusammenhängenden Probleme der Stadtplanung und städtischen Architektur können an dieser Stelle nicht behandelt werden. Vergl. Feux und städtischen Architektur konnen an dieser Stelle GENZMER, Stadtgrundrisse. Ein Rückblick auf ihre geschichtliche En Wreklung, Berlin 1911. des Städtebaus der letzten hundert Jahre, Berlin 1912. - FeLIX GENZMER, Das Haus im Stadtkörper, Berlin 1912 usw. - PAUL ZuCHER, Entwicklung des Stadtbildes, München-Berlin 1929. - Karl GrUber, Die Gestalt der deutschen Stadt, München 1976. - Gerd Albers U.A., Entwicklungslinien im Städtebau. Ideen, Thesen, Aussagen 1875-1945, Düsseldorf 1975. LUDWIG/GruBER (Hrsg.), Die deutsche Stadt im 19. Jahrhundert. Stadtplanung und Baugestaltung im industriellen Zeitalter, München 1974.
Im Rahmen der Rechts- und Verfassungsgeschichte legten Otto Hintze und Fritz Hartung wichtige Zusammenfassungen vor, während Hermann Aubin, Walter Schlesinger, Franz Steinbach, Fritz Rörig, Hektor Amman u. a. die Stadt im Rahmen der Landesgeschichte wiederum vorwiegend im Mittelalter und der frühen Neuzeit weiter verfolgten. In Erich Keysers „Deutschem Städtebuch", das vom 5. Band an später durch Heinz Stoob fortgesetzt wurde, erlebte dieser älteste Zweig der deutschen Urbanistik eine glänzende Kompilation der vorhandenen Erkenntnisse 45. Demgegenüber blieb die sozialwissenschaftlich und mehr theoretisch konzipierte Stadtgeschichte im Gegensatz zu den Vereinigten Staaten stark zurück, was mit der weiterhin bestehenden grundsätzlichen Reserviertheit der deutschen Fachhistoriker gegenüber der Soziologie und Wirtschaftswissenschaft zusammenhing. Obwohl Werner Sombart seine modifizierte Stadtheorie 1931 im Vierkandtschen Handwörterbuch für Soziologie nochmals vorlegte und Gunther Ipsen sie in seiner Bevölkerungslehre aufgriff, der Sombartschüler Arthur Spiethoff sich nochmals prinzipiell über die Rolle von Boden und Wohnung auf dem Mark verbreitete sowie der Geograph Walter Christaller seine vieldiskutierte Theorie der zentralen Orte vortrug, um die ökonomisch-geographischen Gesetzmässigkeit bei der Verteilung und Entwicklung von Siedlungen mit städtischen Funktionen auf die Spur zu kommen, kam es in der engeren Fachhistorie zu keinen ernsthaften Auseinandersetzungen mit diesen Überlegungen ${ }^{46}$. Die Entstehung der modernen Großstadt im 19. Jahrhundert wurde weiterhin, sieht man von Gelegenheitsarbeiten vornehmlich der Lokal- und Kulturgeschichte $a b^{47}$, den Nachbardisziplinen überlassen, vor allem der emporblühenden Bevölkerungswissenschaft, wo Paul Mombert, Helmut Haufe und der später nach Amerika ausgewanderte Tönnies-Schüler Rudolf Heberle ihre bedeutenden Monographien vorlegten ${ }^{48}$. Die Idyllisierung der agrarisch-mittelständischen Welt im Dritten Reich und die Unterdrückung früherer stadtsoziologischer Ansätze zugünsten sozialdarwinistischer und sozialbiologischer

${ }^{45}$ Erich Keyser (Hrsg.), Deutsches Städtebuch. Handbuch städtischer Geschichte, 5 Bde. Stuttgart 1939ff (Bde. V. $1+2$ hrsg. von Heinz StOOB). - OtTo HintZE, Staat und Verfassung. Gesammelte Abhandlungen zur Allgemeinen Verfassungsgeschichte, hrsg. von Gerhard Oestreich, 3. durchges. u. erw. Aufl., 3 Bde., Göttingen 1970. - FriTZ HARTUnG, Deutsche Verfassungsgeschichte vom 15. Jahrhundert bis zur Gegenwart, Leipzig 1914 (9. Aufl. Stuttgart 1969).

${ }^{46}$ Werner Sombart, Art. „Städtische Siedlungen, Stadt“, in: A. Vierkandt (Hrsg.), Handwörterbuch der Soziologie, Leipzig 1931. - GUNTHER IPSEN, Bevölkerungslehre, in: HandWorterbuch des Grenz- und Aulandeutsch zentralen Orte in Süddeutschland, Jena 1933. An 1934. (a)

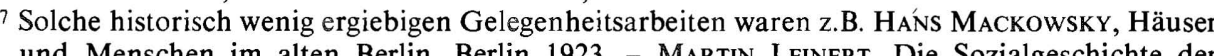
Gerialgeschichte de Zroißstadt, Hamburg 1925. - H. J. G. GATTINEAU, Verstädterung und Arbeiter-Herrschaft, in : . - L. LENZ, Die Mietskaserne in Köln, Bonn-Leipzig 1930. Interessanter erscheint dagegen der Beitrag von K. OLBRICHT, Gedanken zur Entwicklungs-
geschichte der Großstadt, in: Festschrift für Alfred Philippson, 1930, S. 166ff. - Städtisches geschichte der Großstadt, in: Festschrift für Alfred Philippson, 1930, S. 166ff. - Städtisches Bd. 2: Vom 16. Jahrhundert bis zur Gegenwart, Freiburg 1930. - KURT GEBAUER, Deutsche Kulturgeschichte der Neuzeit. Vom Ende des 18. Jahrhunderts bis zur Gegenwart, Berlin 1932. 48 Paul Mombert, Bevölkerungslehre, Jena 1929. - Helmut Haufe, Die Bevölkerung Europas Stadt und Land im 19. und 20. Jahrhundert, Berlin 1936. - Rudolf HeberLe/Fritz MeYer, Die Großstädte im Strom der Binnenwanderung, Leipzig 1937. 
Theorien haben zu dieser Vernachlässigung der historischen Großstadtforschung offensichtlich beigetragen ${ }^{49}$. Die richtungsweisende Zusammenfassung der historisch-sozialwissenschaftlichen Erkenntnisse über den Vorgang der Verstädterung in Louis Wirths Abhandlung "Urbanism as a Way of Life" 1938 blieb auf deutscher Seite, sieht man von Willy Hellpachs kulturpsychologischer Analyse des Großstadtmenschen einmal ab, zwischen 1933 und 1945 ohne Parallele und Widerhall 50.

Wirths Aufsatz ist bis heute ein weiterer großer Markstein in der Urbanistik geblieben, so daß seine Grundgedanken hier wenigstens mit einigen Strichen skizziert werden müssen ${ }^{51}$. Der amerikanische Gelehrte sah es ähnlich wie Georg Simmel zunächst als Aufgabe an, diejenigen Formen gesellschaftlicher Aktion und Organisation herauszufinden, deren Auftreten in relativ dauerhaften, dicht bevölkerten Siedlungen mit einer großen Anzahl heterogener Individuen regelmäßig zu beobachten ist. Die moderne Stadt wird bei ihm durch die Bevölkerungsgröße, die soziale Differenzierung, die Bevölkerungsdichte pro Flächeneinheit und die Konstanz der mittleren Wohndauer bzw. das Ausmaß der sozialen Fluktuation charakterisiert. Auf Bücher, Sombart und Max Weber sich stützend nimmt Wirth zunächst eine Arbeitsteilung zwischen Stadt und ihrem Umland an: Die Stadt ist wenigstens partiell auf agrare Überschüsse der ländlichen Umgebung angewiesen, und diese wird wiederum im Austausch mit städtischen Gewerbeprodukten versorgt. Mit steigender Einwohnerzahl wächst in der Stadt die Zahl der Berufe, die Differenzierung des kulturell-geistigen Lebens und die interne soziale Segregation. Die sich dadurch ergebenden neuen Ideen und Lebensweisen erlebt der Stadtbewohner als stimulierende Außenreize, die er nur durch eine gewisse Auswahl noch verarbeiten kann. Je mehr die urbane Population wächst, um so weniger können sich die Menschen noch gegenseitig kennenlernen, so daß die Blickkontakte als Kommunikation überwiegen. Infolge der Zuwanderung von Bevölkerungsgruppen verschiedenster Art wachsen auch die ethnischen und altersmässigen Unterschiede. Ähnlich wie Simmel weist Wirth darauf hin, daß bei zunehmender physischer Nähe die soziale Distanz wächst. Es bildet sich eine Segmentierung gesellschaftlicher Bindungen heraus, d.h. die Stadtbewohner treten sich weniger als Individuen, sondern mehr in sozialen Rollen gegenüber. Aus personalen Beziehungen werden käufliche Waren und Dienstleistungen. Diese sind aber notwendigerweise an einer möglichst breiten, anonymen Nachfrage interessiert und müssen spezielle Interessen vernach-

49 Vergl. etwa die Arbeit des bekannten nationalsozialistischen Rasseforschers H. F. K. GüNTER, Die Verstädterung. Ihre Gefahren für Volk und Staat vom Standpunkte der Lebensforschung
und Gesellschaftswissenschaft, Leipzig-Berlin 1934. - Vergl. ferner GEORG SCHWEILER, Großstadt und Volkstum, Münster 1933. - B. DE RUDDER/F. LINKE (Hrsg.), Biologie der Großstadt, Dresden-Leipzig 1940.

50 Lours WIRTH, Urbanism as a way of life. In: American Journal of Sociology, vol. 44 (1938), pp. 1-24. Abdruck in: KARL GUSTAV SPECHT (Hrsg.), Soziologische Forschung in unserer Zeit. Festschrift für Ludwig von Wiese, Köln-Opladen 1951, S. 320-335. - ULFERT HERLYN (Hrsg.),
Stadt- und Sozialstruktur Stadt- und Sozialstruktur, München 1974.
Großstadt, Stuttgart 1939 (2. Aufl. 1952)

51 Vergl. C. S. FisHer, Urbanism as a way of Life. A Review and a Agenda. In: Sociological Methods and Analysis, vol. 1 (1972). Die folgenden Ausführungen über Louis Wirth stützen sich auf JÜRGEN FRIEDRICHS, Stadtanalyse. Soziale und räumliche Organisation der Gesellschaft, Reinbek 1977, S. 21-22. lässigen. Wirth sieht damit in der modernen Großstadt prinzipiell deperlassigen. Organisation der Stadt. Die mitmenschlichen Beziehungen werden gegenüber dem früheren Land- oder Kleinstadtleben oberflächlicher, anonym gleichförmiger und schneller veränderlich. Auf der anderen Seite gewinnt der Städter aber auch einen größeren individuellen Freiheitsraum und kann sich traditionalen Kontrollen und Handlungszwängen entziehen, die für kleinere Wohngemeinden charakteristisch sind. Man kann hier Wirths Gedankengang verlängernd hinzusetzen: Die großen Stadtwanderungen des 19. Jahrhunderts müssen neben der Suche nach einem höheren materiellen Lebensstandard hier ihr Hauptmotiv gehabt haben. Persönliche Selbstdarstellungen, überlieferte Tugendmuster und persönliche aktive Teilhabe am politischen Gemeinwesen treten demgegenüber zurück. Da das Individuum seine Interessen in der Großstadt allein nur noch schwer durchsetzen kann, muß es sich notwendigerweise zu neuen Interessenverbänden zusammenschließen, wo man zusätzliche neue Statuspositionen und gesellschaftliche Aufstiegsmöglichkeiten offeriert. Wirth nimmt generell an Simmel sich anlehnend an, daß die Größe und Heterogenität einer urbanen Bevölkerung die entscheidenden Ursachen dafür sind, daß die informalen Kontrollen durch formale bzw. mehr symbolische Zwänge ersetzt werden. So tritt an die Stelle eines gewohnheitsrechtlich oder religiös verankerten Gebots auf dem Dorf die Uhr, die Verkehrsampel oder die Vereinssatzung. Die neuen städtischen Institutionen und Kontrollmechanismen erfassen aber jeweils nur noch Teilbereiche der menschlichen Persönlichkeit, die soziale Integration wird gegenüber früher geringer. Die verminderte gesellschaftliche Bindung verursacht psychische Störungen, die in gesteigerten Selbstmord- und Kriminalitätsraten sowie anderen „abweichenden" Verhaltensweisen zum Ausdruck kommen. Die relativ hohe Bevölkerungsdichte in der Stadt fördert die soziale Segregation, d.h. sozial homogene Gruppen und Schichten ziehen gern zusammen und sondern sich in bestimmten Stadtvierteln ab, so daß dann ein besonderes Lebensmilieu entstehen kann. Im übrigen bewirkt nach Wirth die Kombination von physischer Nähe und psychischer Entfernung den Zug zur allgemeinen Konkurrenz und Ausnutzung gesellschaftlicher Herrschaftspositionen. Deutlich sind bei Wirths Thesen frühere Gedankengänge von Simmel, Sombart und Max Weber zu erkennen.

Nach dem Zweiten Weltkrieg zeigten sich unter dem Eindruck der weiter vorangeschrittenen angelsächsischen und französischen Sozialgeschichte erneut Versuche, sozialwissenschaftliche Methoden und Fragestellungen mit der traditionellen Stadtgeschichte zu verbinden. Unter dem Einfluß der stadtsoziologischen Forschungen von Elisabeth Pfeil, Gunther Ipsen, Rainer Mackensen und Renate Mayntz, der industriell-volkskundlichen Studien Wilhelm Brepohls sowie Werner Conzes Konzept einer strukturgeschichtlichen $\mathrm{Be}$ trachtung legte Wolfgang Köllmann seine für die weitere Urbanisierungsforschung neue Wege weisende „Sozialgeschichte Barmens im 19. Jahrhun-

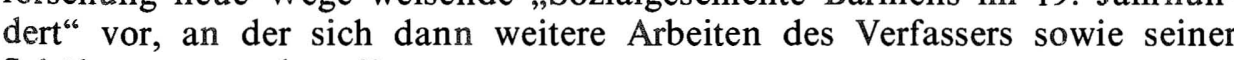
Schüler emporrankten 52 .

52 ElISABeth PFeIL, Großstadtforschung, Bremen-Horn 1950 (2. neubearb. Aufl. Hannover 1972). - Dies., Soziologie der Großstadt, in: ARNOLD GeHLEN/HELMUT SCHELSKY (Hrsg.), Soziologie,
Düsseldorf 1955. - GunTHER IPSEN, Art. „Stadt (IV) Neuzeit“, in: Handwörterbuch der 
Helmut Croon und Otto Büsch versuchten zur gleichen Zeit Anfang der sechziger Jahre, von Heinrich Heffters „Geschichte der Selbstverwaltung im 19. Jahrhundert" ausgehend, in der kommunalen Verwaltungsgeschichte den alten ideen- und institutionengeschichtlichen Rahmen zu durchstoßen 53. Eine Reihe jüngerer Stadthistoriker wie Wolfgang Hofmann, Dieter Rebentisch,' Jürgen Reulecke, Horst Matzerath, Ingrid Thienel, Wolfgang Krabbe und Karlbernhard Jaspers ist seitdem mit neuen Studien gefolgt und hat der Geschichte der Verstädterung neue Lichter der Erkenntnis aufgesetzt ${ }^{54}$. Es ist

Sozialwissenschaften, 9, 1956, Sp. 756-800. - Ders., Verstädterung, in: P. VOGLER/E. KüHN, Medizin und Städtebau, München-Wien 1957, S. 302-317. - R. MACKENSEN U.A., Daseinsformen der Großstadt, Tubingen 1959. - RENATE MAYNTZ, Soziale Schichtung und sozialer Wandel von der agraren zur industriellen Daseinsform dargestellt am Ruhrgebiet, Tübingen 1957. - Dns, Der Aufbau des Ruhrvolkes im Zuge der Ost-West-Wanderung Recklinghausen 1957. - DeRS., Der Aufbau des Ruhrvolkes im Zuge der Ost-West-Wanderung, Recklinghausen 1948. - WoLFGANG KöllmaNN, Sozialgeschichte der Stadt Barmen im 19. Jahrhundert, 1960, S. 25-40. Vergl. ferner: DeRS., Verstadterung, in: Der Mensch in der Großstadt, Stuttgart Vierteljahresschrift für Sozial- und Wirtschaftsgeschichte, 46, 1959, S. 45-70. - DERS., Binnenwanderung und Bevölkerungsstrukturen der Ruhrgebietsgroßstädte im Jahre 1907, in: Soziale Welt, 9, 1958, S. 219-233. - Ders., The Process of Urbanization in Germany at the Height of the Industrialization Period, in: Journal of Contemporary History, 4, 1969, pp. 59-76. 53 Helmut Croon/Kurt UtermanN, Zeche und Gemeinde, Tübingen 1958. - Ders., Forschungsprobleme der neueren Stadtgeschichte, in: Blätter für deutsche Landesgeschichte, 105, 1969, S. 14-26. - DeRS., Zur Entwicklung der Städte im 19. und 20. Jahrhundert, in: Studium generale, 9, 1963, S. 565-575. - DeRs., Methoden zur Erfassung der gemeindlichen Sozialgeschichte des 19. und 20. Jahrhunderts. Erfahrungen aus sozialgeschichtlichen Forschungen im Ruhrgebiet, in: Westfälische Forschungen, 8, 1955, S. 139-149. - DeRs., Studien zur Sozial- und Siedlungsgeschichte der Stadt Bochum, in: Bochum und das mittlere Ruhrgebiet. Festschrift zum 35. Deutschen Geographentag, hrsg. von der Gesellschaft für Geographie und Geologie Bochum, Paderborn 1965. - Oro Busch, Geschichte der Berliner Kommunal-

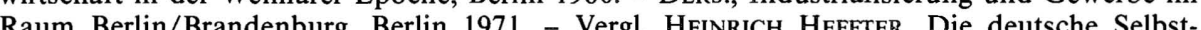
verwaltung im 19. Jahrhundert, Stuttgart 1950 (2. erw. Aufl. Stuttgart 1969).

54 WOLFGANG HOFMANN, Die beiden Ursprünge der modernen Raumordnung und Lebensplanung in den Jahren 1871-1914, in: Gerhard Fehl und Juan Rodriguez-Lores (Hrsg.), Städtebau um die Jahrhundertwende, in: Politik und Planung 10,1980, S. 222-242 - Drss.), Städtebau um Stadtverordneten. Ein Beitrag zur bürgerlichen Selbstverwaltung und sozialem Wandel 1850 1914. Lübeck-Hamburg 1964. - DIETER REBENTISCH, Raumordnung und Regionalplanung im Rhein-Main-Gebiet, in: Hessisches Jahrbuch für Landesgeschichte, 25, 1975, S. 307-339. Ders., Städte und Monopol. Privatwirtschaftliches Ferngas und kommunale Verbundwirtschaft in der Weimarer Republik, in: Zeitschrift für Stadtgeschichte, Stadtsoziologie und Denkmalpflege, 3, 1976, S. 38-80. - DerS., Ludwig Landmann, Frankfurter Oberbürgermeister in der Weimarer Republik, Wiesbaden 1975. - DERS., Kommunalpolitik, Konjunktur und Wers.), Verwaltungsgeschichte, Berlin 1977, S. 107-157. - Jürgen ReulecKe (Hrsg.), Die deutsche Stadt im Industriezeitalter, Wuppertal 1978. - HoRST MATZERATH, Urbanisierung in Preußen (18151914). Habilitationsschrift der Freien Universität Berlin, Masch. Schr. Berlin 1980. - Ders./ KINICHI OGURA, Moderne Verstädterung in Deutschland und Japan, in: Zeitschrift für Stadtgeschichte, Stadtsoziologie und Denkmalpflege, 2, 1975, S. 228-253. - Ders., Von der Stadt zur Gemeinde. Zur Entwicklung des rechtlichen Stadtbegriffs im 19. Jahrhundert, in: Archiv fur Kommunalwissenschaften, 13, 1974, S. 17-46. - INGRID THIENEL, Städtewachstum im

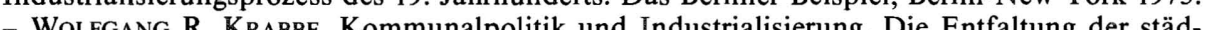
tischen Leistun. Kanaltung in Deutschland bis zum Ersten Weltkrieg dargestelt am Beispie tischen Leistungsverwaltung in Deutschland bis zum Ersten Weltkrieg, dargestellt am Beispiel Schr 1977. nicht notwendig, auf alle diese leicht erreichbaren bekannten Forschungen hier näher einzugehen. Hans Herzfelds bewegte Klage aus der Mitte der sechziger Jahre, die deutschen Historiker hätten die "grüne Weide“ anziehender Themen bei der Verstädterung Deutschlands bisher vernachlässigt, ist damit allerdings noch nicht gegenstandslos geworden 55 . Es darf nämlich nicht übersehen werden, daß die Majorität der bedeutenden Stadtgeschichtsforscher mit Rang und Namen immer noch dem frühen Städtewesen im Mittelalter und der Neuzeit treu geblieben ist, wie etwa die Arbeiten von Fritz Rörig, Hans Planitz, Theodor Mayer, Walter Schlesinger, Erich Maschke, Edith Ennen und Heinz Stoob, aber auch von Karl-Heinz Blaschke, Carl Czok, und Bernhard Toepfer beweisen 56 .

Es läßt sich ferner nicht übersehen, daß das Ende der sechziger Jahre eine gewisse Zäsur in der historischen Urbanisierungsforschung gebracht hat: 1968/69 wurde durch die Amerikaner Stephen Thernstrom und Richard Sennett der Begriff der „New Urban History“ kreiert, mit dem sich folgende Ziele verbinden:

1. Betonte quantitative statt narrative Darstellung.

2. Verbindung historischer Daten mit soziologischer Theorie.

3. Suche nach urbanisierungsrelevanten Epocheneinschnitten.

4. Möglichst Ersatz der traditionellen Städtemonographien durch Vergleich von Städten oder sogar Städtesystemen.

5. Auswertung von Alltagserfahrungen einfacher Menschen für die Stadtgeschichte.

Die Vertreter der „alten Stadtgeschichte“ haben natürlich sofort eingewandt, daß auch sie quantitative Methoden und Quellen benutzt hätten und bei der Neuen Stadtgeschichte" viele Probleme unberücksichtigt bleiben, die sich nicht zahlenmässig messen lassen. Es wurde aber zugegeben, daß hier z.T. Fragen angegangen wurden, die von der herkömmlichen, stark ideen- und institutionengeschichtlich orientierten Stadtgeschichte nicht zureichend erfaßt werden. Der Schwerpunkt der neuen Forschung über die Geschichte der Verstädterung liegt eindeutig bei den demographischen Aspekten (Größe, Zusammensetzung, Verteilung der Bevölkerung in den Städten), bei den

${ }_{55}$ Hans HerzFeld, Leistungen und Aufgaben der Kommunalgeschichte, In: W. Haus (Hrsg.), Kommunalwissenschaftliche Forschung, Stuttgart u.a. 1966. - Ders., Aufgaben der Geschichtswissenschaft im Bereich der Kommunalwissenschaften, in: Archiv für Kommunalmodernen Stadtgeschichte, in: Ebd., 12, 1975, S. 1-21

${ }^{56}$ EDITH ENNEN, Die europäische Stadt des Mittelalters, Göttingen 1972. - W. BEsCH U.A. (Hrsg.), Die Stadt in der europäischen Geschichte, Festschrift für Edith Ennen, Bonn 1972. - WALTER SCHLESINGER, Burg und Stadt, in: Verfassungs- und Landesgeschichte, Festschrift für Th. Mayer, Bd. 1, Lindau-Konstanz 1954. - Hans Planitz, Die deutsche Stadt im Mittelalter, Köln 1954. - FrITZ RörIG, Die europäische Stadt und die Kultur des Bürgertums im Mittelalter, Göttingen 1955. - THEODOR MAYER, Studien zu Anfängen des europäischen Städtewesens, Konstanz 1958. - KARL-HeIZ BLASCHKE, Qualität, Quantität und Raumfunktion als Wesensmerkmale der Stadt vom Mittelalter bis zur Gegenwart, in: Jahrbuch für RegionalGeschichte, Lipzig Jena-Berlin (Ost) 1969 . OTTO BOPST (Hrsg), Stadt in der Geschichte, Sismarinen 1977. - Heisz STOOB, Forschungen zum Städtewesen in Europ deschichte, Wien 1970. - BERNARD TOEPFER, Städte und Ständestaat, Berlin-Ost 1980. Vergl ferner ОтнMAR PICKL (Hrsg), Die Stadt in Geschichte und Gegenwart, Graz 1973. - ЕвіCH MasCHKE, Städte und Menschen, Wiesbaden 1980 
ökologischen Fragen (raumzeitliche Dimensionen sozialer Beziehungen in einer Kommune), bei strukturellen Problemen (Organisation des kommunalen Lebens und seiner Bestandteile), sowie den psychosozialen Verhaltensaspekten des Menschen unter dem Einfluß der Urbanisierung 57. Eine solche Sichtweise ist gerade in der amerikanischen Geschichtswissenschaft allerdings nicht ganz neu 58. Im Juni 1971 versammelten sich die ersten Vertreter der "Neuen Stadtgeschichte" in Madison (Wisconsin) zu einer ersten Konferenz, deren Referate von Leo F. Schnore anschließend herausgegeben wurden 59 . Dieser Band machte den Anspruch der neuen Forschungsrichtung noch deutlicher. Auch in der Bundesrepublik ist die „Neue Stadtgeschichte“, wie die Veröffentlichungen der QUANTUM-Gruppe zeigen, von den Historikern jetzt rezepiert worden: Von 496 Projekten der quantitativ historisch-sozialwissenschaftlichen Richtung im Wintersemester $1977 / 78$ beschäftigten sich immerhin schon 63 mit den Methoden dieser modernen Stadtgeschichte. Der Einsatz der elektronischen Datenverarbeitung und multivariaten Analyseverfahren haben an diesem Aufschwung mitgewirkt 60 .

$$
* * *
$$

Welche Hauptprobleme gibt es in der gegenwärtigen historischen Urbanisierungsforschung? An erster Stelle ist die ungenügende Klärung des Begriffes Verstädterung zu nennen. Im Mittelpunkt aller älteren Definitionsbemühungen stand der Prozeß des historischen Städtewachstums seit dem 19. Jahrhundert, die Verlagerung des Bevölkerungsgewichts vom agraren auf den urbanen Bereich, von der kleinen zu großen Wohngemeinde. Der Prozentsatz der in der Städten lebenden Menschen wird hier als Maßstab für das Wirtschaftswachstum bzw. den erfolgten sozialen Wandel, für Industrialisierung und Modernisierung und sogar für die erreichte Zivilisations- bzw.

57 Stephan Thernstrom/Richard Sennett (eds.), Nineteenth Century Cities: Essays in New Urban History, New Haven-London 1969

${ }^{8}$ So hat Richard Hofstadter in seiner Betrachtung über Geschichte und Soziologie in den USA darauf aufmerksam gemacht, daß schon nach dem 1. Weltkrieg der an der ColumbiaUniversität in New York lehrende Historiker James H. Robins eine "New History“ forderte, die alle politischen Ereignisse betont von der wirtschaftlichen und gesellschaftlichen Seite her sehen sollte, insbesondere von der Umgebung und dem Milieu her, wobei die Erfahrungen einfacher Menschen und die Methoden der Soziologie genutzt werden sollten. Der Einfluß des damals modischen Behaviorismus und der einsetzenden amerikanischen Urban Sociology wird hier deutlich. Die New Urban History als Pendant zu einer New Economic History und New Vorgl H. P.ory kann als Verlanngerung solcher wissenschaftlichen Traditionen gesehen werden. York 1968, p. 8 .

59 LeO F. SCHNOnE (ed,) The New Urban History, Quantitative Explorations by American He. SCHNORE (ed.), The New Urban History, Quantitative Explorations by American History. In: Daedalus, vol. 100 (1971) pp. 359-375. - THEO, R HerSHBe 0 The New Urban History: Toward a Interdisciplinary History of City. In: Journal of Urban History, vol.5 (1978), pp. 1-40. - JoYce AschenBRenNer-Lloyd/R. Collins (eds), Process of Urbanism: A Mulpp. 1-40. - JOYCE AsCHENBRENNER-LLOYD/R. COLLINS (eds), Process of Urbanism: A MulUrban History ist PETER R. KNIGHT, The Plain People of Boston, 1830-1860. A Study in City Growth, New York 1971.

60 WILHELM SCHRÖDER (Hrsg.), Moderne Stadtgeschichte, Stuttgart 1979.
Kulturhöhe einer Gesellschaft gesehen. Mit einer solchen Betrachtung bewegt man sich freilich auf unsicherem Boden: Läßt sich tatsächlich eine Gesellschaft oder ein Staat bereits als "verstädtert" bezeichnen, wenn etwa ein Drittel oder die Hälfte seiner Bewohner in den Städten leben? Zwar hat schon 1887 der internationale Statistikerkongreß festgelegt, daß Städte mit mehr als 100.000 Einwohnern als "Großstädte" bezeichnet werden sollen. Auch die deutsche Statistik hat im Anschluß daran Mittelstädte mit 20-100.000, Kleinstädte mit 5.000-20.000 und Landstädte mit 3.000-5.000 Bewohnern davon unterschieden. Es wird aber bei dieser rein zahlenmäßigen Einteilung nicht deutlich, wo die entscheidenden Schwellenwerte für eine Verstädterung beginnen. Eine oberdeutsche Handelsstadt im 16. Jahrhundert mit 15.000 Einwohnern war für die damalige Zeit sicherlich eine Großstadt". Sie überragte in ihrer weitreichenden Bedeutung eine moderne Agglomeration von 100.000 Menschen wie beispielsweise Wanne-Eickel. Gleitende Bemessungsgrenzen, die daraufhin vorgeschlagen wurden, können leider dieses Problem nicht lösen, da sich offenbar mit der städtischen Quantität auch die städtische Qualität ändert 61

Das Unbehagen an dem Großstadtbegriff hat dazu geführt, dynamische Strukturveränderungen wirtschaftlich-sozialer Art zur Bestimmung der Verstädterung mit heranzuziehen ${ }^{62}$. Unter anderem ist an Jean Fourasties wirtschaftliche Wachstumstheorie sich anlehnend auf die sich arbeitsteilig verfeinernde Entwicklung von Gewerben und Dienstleistungen in den Städten hingewiesen worden. Auch Werner Sombart und Max Weber hatten um 1900 bei ihrer ersten Stadttheorie bekanntlich auf die Arbeitsteilung von Stadt und Land und auf diesen Punkt aufmerksam gemacht. Die Städte erscheinen hier als besondere Quellen ökonomischer und sozialer Innovationen. Dabei erhebt sich allerdings wiederum die Frage, ob man hier nicht nur bestimmte, einmalig ablaufende historische Prozesse erfaßt und ob die Stadt am Ende dieses 20. Jahrhunderts immer noch eine Vorbedingung für solche weitreichenden Strukturveränderungen darstellt. Es könnte auch durchaus sein, daß die Rolle der Stadt in einer bereits verstädterten Gesellschaft überschätzt wird. Daran läßt sich die Frage knüpfen, welche Kontinuitäten eigentlich zwischen antiken, mittelalterlichen, absolutistisch-merkantilistischen, frühindustriellen und modernen Städten bestehen und inwieweit sich der Begriff "Stadt“ in seinem Kern verändert hat. Die vielen Bücher mit dem Titel „Die Stadt in Vergangenheit und Gegenwart" suggerieren die Konstanz eines Sachverhaltes, der so nicht gegeben ist. Die moderne Begriffsforschung ist mit anderen Worten

${ }^{61}$ Gleitende Bemessungsgrenzen für die Definition von Stadtgrößen sehen vor, daß eine Stadt um 1600 ab 15.000 , um 1790 ab 20.000, um 1840 ab 40.000 und um 1939 ab 100.000 Bewohnern als Großst adt angesen Verstädterung unterschlagen werden. Eine spätmittlalterliche Stadt ist, auch wenn sie grössenmäßig im gleichen Verhältnis zu anderen Städten und zur Landbevölkerung wie eine heutige Großstadt dasteht, noch lange keine moderne Metropole. Bestimmte städtische Qualifikationen und Charakteristika sind, wie die Stadtheorien von Simmel und Wirth zeigen, auch an bestimmte absolute Größenverhältnisse gebunden 62 NeILS ANDERSON (ed.), Urbanism and Urbanization, Leiden 1964.-LeONARD ReISMANN, The
Urban Process. Cities in Industrial Societies, London 1964. - ERIC E. LAMPARD, Urbanization and Social Change. On Scope and Relevance in Urban History. In: OsCaR HandLin/JoHN BURCHARD (eds.), The Historian and the City, Cambridge 1963, pp. 225-247. 
voll in die Urbanisierungsforschung einzubeziehen. Das Problem kann hier nur angedeutet werden und verdiente eine ausführlichere Behandlung.

Das erregendste Problem ist sicherlich der mögliche Zusammenhang zwischen Industrialisierung und Urbanisierung. Die wissenschaftlichen Ansichten gehen hier weit auseinander: Einmal wird das wirtschaftliche „Take-off into sustained growth" (W. W. Rostow) als notwendige Vorbedingung einer Verstädterung angesehen, zum anderen nimmt man nur gewisse Beschleunigungseffekte an, die vom wirtschaftlichen Wachstum auf die städtische Entwicklung ausstrahlen. Es wird aber auch von anderen die zeitliche Parallelität beider Prozesse betont oder aber ganz umgekehrt die Urbanisierung als Prämisse für die einsetzende Industrialisierung betrachtet. Schließlich gibt es noch Forscher, die nur ganz lose Zusammenhänge sehen oder sogar jede Koinzidenz leugnen, weil es sich um zwei prinzipiell autonom verlaufende Prozesse handelt ${ }^{63}$. In der Urbanisierungstheorie ist in diesem Zusammenhang bekanntlich wiederholt darauf verwiesen worden, daß die Einführung der Geldwirtschaft die räumliche wie soziale Mobilität der ländlichen Bewohner ungemein befördere und die Stadtbildung dadurch begünstigt habe. Die Städte waren erste Zentren der Geldwirtschaft. Aber dies mußte später nicht notwendigerweise immer zur Industriebildung im engeren Sinn führen. Es gab daneben offenbar viele andere "Städtefüller“, die nicht einmal indirekt im Rahmen des Gesetzes vom doppelten Stellenwert mit der gewerblichen Industrie zu tun hatten. Der Verzehr von Grundrenten wie auch die Kapitalakkumulation auf engem Siedlungsraum lösten eine erweiterte Nachfrage nach Gütern und Dienstleistungen aus. Ökonomisch entscheidend für Stadtgründungen wie für Stadtwachstum war offensichtlich, wieviel Einkommen pro Kopf des Bewohners ausgegeben werden konnte. Bei der Beantwortung dieser Fragen ist auf jeden Fall auf die historisch gewachsenen Stadttypen hinzuweisen, die ganz verschiedene ökonomische, soziale und politisch-kulturelle Funktionen hatten. So gab es im 19. Jahrhundert städtische $\mathrm{Ag}$ glomerationen, die praktisch eine einzige große Fabrik waren und bei denen der Einfluß der Industrie auf das städtische Wachstum evident war. Ähnliches läßt sich vom Einfluß des Seeverkehrs auf Hafenstädte oder des Bergbaus auf Bergstädte sagen. Daneben gab es aber auch fast reine Residenz-, Verwaltungs- und Universitätsstädte, ferner Festungs- bzw. Garnisons- sowie Pensionärs- und Kurstädte. Wenngleich die Mischformen und Überlappungen hier dominieren, so läßt sich doch sagen, daß die Städte in ihren ökonomischen Funktionen vielfach überhaupt nicht vergleichbar waren. Hinter dem Idealtypus „Stadt im Industriezeitalter“ verbirgt sich viel Heterogenes. Die bekannte These von der Industrie als „Städtefüller“ übersieht jedenfalls, $\mathrm{da} ß$ in den Industriestädten viele Bewohner nichts direkt mit der Industrie zu tun hatten.

${ }^{63}$ BERT F. HoseutTz, The Role of Cities in the Economic Growth of Underdeveloped Countries. In: DERS., Sociological Aspects of Economic Growth, Glencoe, Ill., 1960, p. 159. - PHILIP M.
HAUSER, The Social, Economic and Technical Problems of Rapid Urbanization. In: BERT F. HAUSER, The Social, Economic and Technical Problems of Rapid Urbanization. In: BERT F. Lösch, Das Problem einer Wechselwirkung zwischen Bevölkerungs- und Wirtschaftsentwicklung, in: Weltwirtschaftliches Archiv, 48, 1938, S. 455ff. - ERNST WILLEKE, Zur Entstehung und Problematik der Großstadt, in: Soziale Welt, 6, 1955. - WoLfGANG KöLLMANN, Verstädterung, in: Der Mensch in der Großstadt, Stuttgart 1960, S. 28. - F. MerzBaChER, Art. „Stadt. 1. Neuere Geschichte“, in: Staatslexikon, Bd. 7, 6. neubearb. und erw. Aufl. Freiburg 1962, Sp. $631 \mathrm{ff}$.
In der Debatte um den Zusammenhang zwischen Urbanisierung und Industrialisierung ist schließlich noch zu wenig gesehen worden, daß die Verstädterung offenbar durch metaökonomische Faktoren zum Teil entscheidend behindert oder gefördert worden ist. Die vielfach beobachtete Gründung von Doppel- und Konkurrenzstädten in nächster Nachbarschaft hatte wenig mit den ökonomischen Funktionen, dagegen viel mit den natürlich-geographischen Verhältnissen und vor allem mit Grenzziehungen zu tun. Infolge bestimmter politischer Fakten konnte es auch zu städtischen Verkümmerungen oder sogar Entstädterungen kommen.

Nach über einhundert Jahren Urbanisierung läßt sich erkennen, daß frühere Untersuchungen viel zu einseitig das Problem der Verstädterung mit einer destruktiven „Landflucht“ (Peter Quante) gleichgesetzt und diese Bevölkerungsbewegung einseitig dramatisiert haben. Über Ausmaß und Richtung der Stadtwanderungen bestanden lange keine quantitativ wie qualitativ zutreffenden Vorstellungen. So konnte man nicht angeben, welche ländlichen Bevölkerungsteile wohin abwanderten und wo auf dem Lande wirklich Entvölkerungserscheinungen auftraten. Erst neuere historische Mobilitätsstudien geben erste Einsichten über die komplizierten selektiven Wanderungsbewegungen, die sich offenbar in viele Richtungen bewegten und auch ein hohes $\mathrm{Ma} \beta$ an Stadt-Land-Pendlern und Rückwanderern umfaßten. Noch gar nich untersucht sind die tatsächlichen Rückwirkungen der Verstädterung auf die ländlichen Daseinsverhältnisse wie die dort möglichen Gegenbewegungen (Counter-Urbanization). Schließlich ist daran zu denken, daß es neben der bereits länger verstädterten Bevölkerung in den Stadtkernen an den Grenzen von Stadt und Land zahlreiche Übergangsformen der Verstädterung gegeben hat. Es kann mit Recht vermutet werden, daß sich die mit der Verstädterung einhergehenden Prozesse der sozialen Desorganisation besonders kraß in den großstädtischen Vororten darstellten. Da sich die Urbanisierung nicht so seh in der bloßen Bevölkerungsanhäufung als vielmehr in der Ausbildung eines besonderen städtischen Lebensstils äußerte, muß nicht nur an die demographischen, sondern auch an die kulturellen Konzepte der Verstädterung gedacht werden, wie Eric E. Lampard mit Recht betont hat 64 . Im Grunde umfaßt die Urbanisierung die Totalität aller sozialen Handlungen, Normen und Institutionen. Der Vorgang der Verstädterung greift regelmäßig auf die Dauer über die Stadtgrenzen hinaus: Urbane Verhaltensweisen beeinflussen die Umgebung und durchdringen dann das ganze Land, so daß sich die alten Unterschiede einebnen, was mit dem treffenden Begriff „Rurbanization" bezeichnet worden ist. Soziale und ethisch-kulturelle Unterschiede zwischen einzelnen Stadtvierteln können dann unter Umständen gravierender als der alte Stadt-Land-Gegensatz werden. Inzwischen gibt es kaum noch Zweifel daran, daß in den Industrieländern die städtischen Verhaltensmuster ubiquitär geworden sind. Henri Lefebvre hat daher nicht zu Unrecht die Frage aufgeworfen, ob die postindustrielle Gesellschaft nicht insgesamt als ,verstädterte Gesellschaft" bezeichnet werden sollte 65. Faßt man die wichtigsten Erklä-

${ }^{64}$ ERIC E. LAMPard, Historical Aspects of Urbanization. In: Philipp E. Hauser/Leo F. SCHNoRe (eds.), The Study of Urbanization, New York-London-Sidney 1965, pp. 519.

${ }^{65}$ HeNRI Lefebvre, Die Revolution der Städte (Übers. aus dem Franz.), München 1972. 
rungsansätze für die Urbanisierung zusammen, dann erhält man folgende Bestimmungsmerkmale:

1. Umschichtung von primär ländlicher auf vorwiegend städtische Bevölkerung und gleichzeitig allgemeines Bevölkerungswachstum.

2. Umschichtung des wirtschaftlichen Schwergewichts vom agraren auf den gewerblichen und dienstleistenden Sektor.

3. Herausbildung neuer Sozialstrukturen und verstärkte räumliche wie soziale Mobilität.

4. Diffusion urbaner Mentalität auf die Gesamtgesellschaft.

$\mathrm{Zu}$ den kontroversen Problemen der Urbanisierungsforschung gehört schließlich auch die Periodisierungsfrage. Rainer Mackensen hat zwischen feudalen, merkantilistischen, bürgerlichen und industriellen Verstädterungen unterschieden 66 . Hier wird aber offenbar ganz übersehen, daß die Verstädterungen vor 1870 regional zu verschiedenen Zeitpunkten auftraten und die wirtschaftlich-soziale Gesamtstruktur insgesamt wenig tangiert wurde. Der Anteil der städtischen Bevölkerung an der Gesamtpopulation blieb bekanntlich bis zum späten 19. Jahrhundert relativ gering, da unter anderem der Versorgung großer Menschenmassen an einem einzigen Or wegen der vergleichsweise geringen Transportkapazität enge Grenzen gesetzt waren. Zweckmäßiger erscheint die Annahme von drei großen Umbruchphasen:

1. Die vorindustrielle Periode, in der die Städte noch keine große Überschußbevölkerung vom Lande aufnehmen konnten, so daß nur die Auswanderung als Notventil verblieb, wenn Kriege, Seuchen oder Mißernten die Menschen nicht auf natürliche Weise dezimierten. Die räumliche wie gesellschaftliche Mobilität blieb insgesamt gering: die meisten Stadtbewohner waren in Wahrheit "Ackerbürger".

2. Die Phase der extensiven Verstädterung mit rasch wachsender Ausdehnung der Städte wie der Gesamtbevölkerung.

3. Der Übergang zu einer weithin verstädterten Gesellschaft, in der 70. v.H. aller Menschen in der Stadt leben und urbane Verhaltensweisen die Kultur prägen.

Nach Horst Matzeraths Berechnungen über die Urbanisierung in Preußen lassen sich folgende, noch genauere zeitliche Einschnitte ausmachen:

Zwischen 1840/43-1868/71 stieg der Anteil der urbanen Bevölkerung nur auf niedrigem Niveau an, so daß von einer Inkubationsphase der Verstädterung gesprochen werden kann. Der dramatische Aufstieg zur Verstädterung ereignete sich, gemessen an den Bevölkerungsziffern, erst zwischen 1871 und 1910 im Deutschen Reich. In der nachfolgenden Zwischenkriegszeit nahm die Bevölkerungsvermehrung auf relativ hohem Niveau schon wieder ab.

Diese rein quantitative Betrachtung (die an die Zufälligkeiten einer Zensuserhebung gebunden bleibt!) läßt, wie gesagt, alle anderen Wechselwir-

\footnotetext{
${ }^{66}$ In der Periode der feudalen Verstädterung bilden agrarische Überschüsse des Umlandes die Grundlage zur Gründung von Konsumentenstädten. In der nachfolgenden merkantilistische Phase ergeben sich aus dem sich ausbreitenden Fernhandel die Gründe für eine urbane Siedlungsweise. In der folgenden liberalen Ara nach 1800 gibt es infolge von Verwaltungsreformen eine bürgerliche Verstädterung und schließlich eine Urbanisierung unter dem Einfluß der Industrialisierung. Vergl. Rainer Mackensen, Städte in der Statistik, in: W. Pehn (Hrsg.), Die Stadt in der Bundesrepublik, Stuttgart 1974, S. 141.
}

kungen und Strukturverformungen, die unter Umständen anderen Zeitrhythmen folgen, außer acht. Agrarreformen, Gewerbefreiheit und Verkehrsbeschleunigung haben die Verstädterung sicherlich vorangetrieben, doch wurden alle diese wichtigen Innovationsschübe nicht überall und nicht zum gleichen Zeitpunkt in Gang gesetzt. Regionale Differenzierungen der Urbanisierung sind daher unabdingbar, wobei zwischen stadtanziehenden "Pull-Faktoren" und landabstoßenden "Push-Faktoren" unterschieden werden muß. Vom Grundmuster der hier zuletzt aufgezeigten Periodisierung gibt es offenbar viele Abweichungen, was bei den vielen Stadttypen verständlich erscheint. Die regionalen und lokalen Sonderheiten der Urbanisierung, die mit den spezifischen Bau- und Wohnungsmärkten, dem Aufbau der Infrastrukturen sowie der kommunalen Leistungsverwaltung zusammenhängen, sind bis heute noch wenig zusammhängend erforscht. Am wenigsten wissen wir aber über die bei der Periodisierung eingangs erwähnten soziokulturellen Veränderungen, die Ausbildung und Diffusion urbaner Mentalitäten, was sich nur sehr begrenzt quantitativ erfassen läßt.

Bei der Urbanisierungsdebatte stellt sich am Schluß die Fragen nach einer genaueren Bestimmung der Untersuchungsebenen. Objekte können z. B. sein 67

1. Einzelne Stadtbewohner.

2. Formale und informale Gruppen, Schichten und Institutionen von städtischen Gemeinden.

3. Städte als historische Individuen.

4. Städte als Subsysteme einer jeweiligen Gesellschaft.

Nach diesem Schema könnte zwischen internen und externen Funktionen der Städte, d. h. zwischen der Geschichte einer inneren Stadtstruktur und einer äußeren Geschichte der Städte im Rahmen der Gesamtgeschichte gesprochen werden. Aber eine solche Differenzierung zwischen der Entwicklung einer Stadt, die gleichsam eine kleine Welt für sich darstellt und ihren äußeren Beziehungen läßt sich generell nicht durchhalten und ist heuristisch daher von geringem Wert.

Jürgen Friedrichs hat 1977 darauf hingewiesen, die Stadtsoziologie habe sich bisher unfähig erwiesen, die Materialfülle städtischen Daseins systematisierend zu durchdringen und meinte, der Mangel an Theorie hänge mit der unscharfen Trennung von individueller Stadt- und genereller Gesellschaftsanalyse zusammen. Er bezweifelte, daß eine Unterscheidung von Stadt und Land heute noch sinnvoll sei, weil wir bereits in einer weithin verstädterten Gesellschaft leben ${ }^{68}$. Alle Stadtanalysen seien auch Gesellschaftsanalysen und umgekehrt. Gegenstand der Stadtsoziologie sei in Wahrheit nicht die Stadt, sondern das raumbezogene Verhalten des Menschen. Die Stadt sei sozialwissenschaftlich überhaupt kein abgrenzbarer Objektbereich, sondern

67 U. POSECK, Geographische Auswirkungen der Verstädterung als Lebensform. Ein soziologischer Beitrag zur Genese des Städtischen, Diss. Köln 1966. - JOYCE AsCHENBRENNER ET. AL. (eds.), Process of Urbanization: A Multidisciplinary Approach, Den Haag 1978. - RICHARD WADE, An Agenda for Urban History. In: Herbert Bas (ed.), The State of American History, Chicago 1980, pp. 79.

68 J. Friedrichs, Stadtanalyse 1977, S. 14 - Vergl, auch Peter AtTesLander/Bernhard Hamm (Hrsg.), Materialien zur Siedlungssoziologie, Köln 1974 
nur ein Anwendungsgebiet zur Erklärung von Sachverhalten, die sich unter anderem auch in der Stadt nachweisen lassen.

Friedrichs These, alle Stadtforschung heute sei im Grunde Gesellschaftsanalyse, ist für den Stadthistoriker auf den ersten Blick bestechend, weil auch Stadtgeschichte, zugespitzt formuliert, eigentlich niemals etwas anderes gewesen ist als eine Sozialgeschichte von einem besonderen räumlichen Standpunkt aus betrachtet 69 . Dennoch reicht ein solcher sozialökologischer Ansatz nicht aus, um alle Urbanisierungsprobleme zu erklären. Die Analyse der Bevölkerung auf städtischem Gebiet kann ohne Zweifel interessante soziale Segregationen, z. B. das Vorhandensein von Wohlstands- und Armenvierteln oder Wohn- und Industriegebieten als extreme Sondergestaltungen städtischen Lebens sichtbar machen. Auch die Aufdeckung von Nachbarschaftsbeziehungen, die Anordnungen der Wohnungen zu den Arbeitsstätten mit den Pendlerbewegungen, die Bevölkerungsverschiebungen bei City- und Vorortbildungen sind ein dankbares Feld solcher Untersuchungen. Dennoch bleibt die städtische Gemeinde letztlich mehr als eine Ansammlung von Menschen unter bestimmten räumlichen Umweltbedingungen. Sie zeichnet sich nicht zuletzt auch dadurch aus, daß die Integration zum städtischen Bürger über das Medium des politisch-kulturellen Lebens verläuft, weil die Komplexität der sozialen Strukturen für den einzelnen Bewohner nicht überschaubar ist und diese sich auch ständig ändern. Jede Stadt zeichnet sich, wie schon Louis Wirth gezeigt hat, neben spezifischen sozialen und geographischen Merkmalen durch einen besonderen politisch-kulturellen Lebensstil aus. Einer Quantifizierung sind daher bestimmte Grenzen gesetzt. Das Messen von generativem Verhalten, Bevölkerungswachstum, Wanderungen, Agglomerationen und Segregationen führt zur Aufdeckung interessanter Regelmäßigkeiten und Interdependenzen, doch dürfen die Probleme von Recht und Verfassung, Politik und Kultur nicht ausgeblendet werden. Die frühere Stadtgeschichtsforschung hat die Fragestellungen der "Neuen Stadtgeschichte“ $\mathrm{zu}$ wenig berücksichtigt, aber es wäre ebenso verkehrt, auf die Erforschung individuell-geistiger Bezüge, der rechtlich-politischen Verfassung und der kulturellen Mentalität generell zu verzichten. Die nachfolgenden Beiträge der Historiker sind sowohl der einen wie der anderen Richtung verpflichtet. Insgesamt wurde versucht, einen Mittelweg zu finden und möglichst vielen Ansprüchen sowohl methodologisch wie inhaltlich gerecht zu werden. 